\title{
Feynman-Diagrammatic Description of the Asymptotics of the Time Evolution Operator in Quantum Mechanics
}

\author{
THEO JOHNSON-FREYD \\ University of California, Berkeley, USA. e-mail: theojf@math.berkeley.edu
}

Received: 18 April 2010 / Revised: 2 September 2010 / Accepted: 4 September 2010

Published online: 30 September 2010 - (C) The Author(s) 2010. This article is published with open access at Springerlink.com

\begin{abstract}
We describe the "Feynman diagram" approach to nonrelativistic quantum mechanics on $\mathbb{R}^{n}$, with magnetic and potential terms. In particular, for each classical path $\gamma$ connecting points $q_{0}$ and $q_{1}$ in time $t$, we define a formal power series $V_{\gamma}\left(t, q_{0}, q_{1}\right)$ in $\hbar$, given combinatorially by a sum of diagrams that each represent finite-dimensional convergent integrals. We prove that $\exp \left(V_{\gamma}\right)$ satisfies Schrödinger's equation, and explain in what sense the $t \rightarrow 0$ limit approaches the $\delta$ distribution. As such, our construction gives explicitly the full $\hbar \rightarrow 0$ asymptotics of the fundamental solution to Schrödinger's equation in terms of solutions to the corresponding classical system. These results justify the heuristic expansion of Feynman's path integral in diagrams.
\end{abstract}

Mathematics Subject Classification (2010). 81T18, 81S40, 81Q15.

Keywords. quantum mechanics, Feynman diagrams, formal integrals, path integrals, semiclassical asymptotics.

\section{Introduction}

The goal of this paper is to show that the semiclassical description of the evolution operator in quantum mechanics in terms of Feynman diagrams satisfies all the necessary requirements: it satisfies the Schödinger equation and has the correct initial conditions. This description is rooted in Feynman's path integral interpretation of quantum mechanics, first published in [5] (see also [6]), and formal semiclassical arguments. In certain cases, non-perturbative path integrals can be interpreted analytically as Weiner integrals, e.g. $[1,7,10]$. However, for the last half century, physicists have used "formal" perturbative path integrals and Feynman diagrams extensively to make perturbative empirical predictions in theories that do not have adequate mathematical foundations, and there have been similar mathematical applications to the subject of topological quantum field theory [15]. Surprisingly, the diagrammatic methods have not previously been shown to give the correct answers when applied to the one mathematically rigorous nonperturbative theory available, namely nonrelativistic quantum mechanics. We fill that gap. 
In this paper we consider quantum mechanics in $\mathbb{R}^{n}$ with the Hamiltonian being a second-order differential operator. (In [8] we will discuss perturbative quantum mechanics on general Riemannian manifolds.) Recall some basic definitions. Let $B_{1}, \ldots, B_{n}, C: \mathbb{R}^{n} \rightarrow \mathbb{R}$ be smooth functions. ${ }^{1}$ The nonrelativistic Schrödinger operator with electric potential $C$ and magnetic potential $B=\left(B_{1}, \ldots, B_{n}\right)$ is the following linear second-order differential operator on $\mathbb{R}^{n}$ :

$$
\hat{H}=\sum_{j=1}^{n}\left(i \hbar \frac{\partial}{\partial q^{j}}+B_{j}(q)\right)^{2}+C(q)
$$

Solutions to the partial differential equation $\frac{\partial}{\partial t} \psi(t, q)=\frac{1}{i \hbar} \hat{H} \psi(t, q)$ with fixed initial data define a one-parameter family of unitary "time evolution" operators $U(t)$ on $L^{2}\left(\mathbb{R}^{n}\right)$. The kernel of the time evolution operator is a distribution $U\left(t, q_{0}, q_{1}\right)$, satisfying $(U(t) \psi)\left(q_{1}\right)=\int_{\mathbb{R}^{n}} U\left(t, q_{0}, q_{1}\right) \psi\left(q_{0}\right) \mathrm{d} q_{0}$. It is determined by the following initial value problem:

$$
i \hbar \frac{\partial}{\partial t} U\left(t, q_{0}, q_{1}\right)=\hat{H}_{q_{1}}\left[U\left(t, q_{0}, q_{1}\right)\right] \quad U\left(0, q_{0}, q\right)=\delta\left(q-q_{0}\right)
$$

The operator $\hat{H}_{q_{1}}$ acts on the $q_{1}$ variable, leaving $\left(t, q_{0}\right)$ fixed. In this paper we describe explicitly the asymptotics of $U\left(t, q_{0}, q_{1}\right)$ as $\hbar \rightarrow 0$, at least for $\left(t, q_{0}, q_{1}\right)$ in a dense open subset of $\mathbb{R}_{>0} \times \mathbb{R}^{2 n}$.

The asymptotics of $U\left(t, q_{0}, q_{1}\right)$ are known to be given by the Hamilton-Jacobi action and solutions to the transport equation [16]. Feynman's path integral also predicts that the asymptotics are given by a power series where coefficients are parameterized by "Feynman diagrams": each diagram represents a finitedimensional integral, and the coefficients of the power series are the values of these integrals. In this paper we will define this diagrammatic expansion; the integrands depend on the solutions to the corresponding classical mechanical system. We will prove that the Feynman-diagrammatic power series satisfies formal versions of equation (1.0.2): it satisfies the Schrödinger equation as a function valued in formal power series, and as a distribution valued in formal power series approaches the $\delta$ distribution as $t \rightarrow 0$.

In select cases these results are already known. For the quadratic "harmonic oscillator" (the functions $B_{1}, \ldots, B_{n}$ are linear in position, $C$ is quadratic), the path integral is defined directly from Gauss's formula, and agrees with the Schrödinger picture [16]. Feynman's original argument applies when the corresponding classical physics is a perturbation of the free theory, i.e. when $B_{1}, \ldots$, $B_{n}, C$ are infinitesimal quantities. Combining these observations, it is not too difficult to prove that the diagrammatic path integral satisfies Schrödinger's equation

${ }^{1}$ Without much difficulty, these functions can be taken to depend explicitly on an external time parameter, but for notational convenience we do not do so. The function $U\left(t, q_{0}, q_{1}\right)$ that we will discuss should then be a function of two time variables $U\left(t_{0}, t_{1}, q_{0}, q_{1}\right)$, and similar superficial changes must be made throughout this paper. 
for infinitesimal perturbations of the harmonic oscillator. More importantly, Duru and Kleinert [3] apply the path integral approach to calculate the energy spectrum of a particle moving in the Coulomb potential, and compare their approach to the Schrödinger methods. Their methods differ from ours: we disallow singular potentials but consider arbitrary smooth ones, so our results do not apply to the Coulomb potential; moreover, they take advantage of numerous changes of variables (without explicitly checking the coordinate invariance of the path integral) and use the "charge" of the potential well as the perturbation parameter, whereas we define the path integral directly and use Planck's constant $\hbar$ for perturbation. Diagrammatic path integrals do exist in the work of Kleinert and Chervyakov [11] and in the work of DeWitt-Morette [2]; Kleinert and Chervyakov's methods apply directly only to perturbations of free motion, and neither they nor DeWittMorette (who does give an expansion equivalent to ours) check the Schrödinger equation directly, although the result is implicit in their and Duru and Kleinert's approaches.

We summarize the main definitions and results in Section 2. Section 3 has proofs of two key lemmas. The main theorem is proven in Section 4, and in Section 5 we prove the short-time asymptotics.

\section{Statements of the Definitions and Results}

\subsection{PRELIMINARIES}

We begin by establishing some notation. Kronecker's $\delta$-matrix is $\delta_{j}^{i}=1$ if $i=j$ and 0 otherwise. Dirac's $\delta$-function is the distribution defined by $\int_{-\infty}^{\infty} f(x) \delta(x) \mathrm{d} x=$ $f(0)$. Heaviside's step function is:

$$
\Theta(x)=\int_{-\infty}^{x} \delta(t) \mathrm{d} t= \begin{cases}0, & x<0 \\ \frac{1}{2}, & x=0 \\ 1, & x>0\end{cases}
$$

We henceforth adopt Einstein's summation convention: $p_{i} q^{i}=q^{i} p_{i}=\sum_{i=1}^{n} p_{i} q^{i}$. We raise and lower indices as is convenient: $p^{i}=p_{i}$.

DEFINITION 2.1.1. A (piecewise-smooth parameterized) path is a continuous function $\gamma:[0, t] \rightarrow \mathbb{R}^{n}$ such that there exists a decomposition $0=t_{0}<t_{1}<\cdots<t_{n}=t$ with $\left.\gamma\right|_{\left[t_{j}, t_{j+1}\right]}$ smooth for each $j=0, \ldots, n-1$. The duration of a path $\gamma:[0, t] \rightarrow$ $\mathbb{R}^{n}$ is the real number $t$. A based loop is a path $\gamma:[0, t] \rightarrow \mathbb{R}^{n}$ with $\gamma(0)=0=\gamma(t)$. A path $\gamma$ is classical if it satisfies the following nonlinear second-order differential equation (we write $\dot{\gamma}(\tau)=\frac{d}{d \tau} \gamma(\tau)$, etc.):

$$
0=\ddot{\gamma}^{i}(\tau)+\left(\frac{\partial B_{i}}{\partial q^{j}}(\gamma(\tau))-\frac{\partial B_{j}}{\partial q^{i}}(\gamma(\tau))\right) \dot{\gamma}^{j}(\tau)+\frac{\partial C}{\partial q^{i}}(\gamma(\tau))
$$


Let $\gamma, \xi$ be paths of duration $t$, and define the differential operator $\mathcal{D}_{\gamma}$ given by:

$$
\begin{aligned}
\mathcal{D}_{\gamma}[\xi]^{i}(\tau)= & \dddot{\xi}^{i}(\tau)+\left(\frac{\partial B_{i}}{\partial q^{j}}(\gamma(\tau))-\frac{\partial B_{j}}{\partial q^{i}}(\gamma(\tau))\right) \dot{\xi}^{j}(\tau) \\
& +\left(\frac{\partial^{2} B_{i}}{\partial q^{j} \partial q^{k}}(\gamma(\tau)) \dot{\gamma}^{k}(\tau)-\frac{\partial^{2} B_{j}}{\partial q^{i} \partial q^{k}}(\gamma(\tau)) \dot{\gamma}^{k}(\tau)+\frac{\partial^{2} C}{\partial q^{i} \partial q^{j}}(\gamma(\tau))\right) \xi^{j}(\tau)
\end{aligned}
$$

A classical path $\gamma$ of duration $t$ is nondegenerate if the kernel of $\mathcal{D}_{\gamma}$ among based loops of duration $t$ is trivial.

As equation (2.1.2) is nondegenerate second-order, a classical path $\gamma$ is determined by its duration and its initial conditions $(\dot{\gamma}(0), \gamma(0))$. Thus the space of classical paths is naturally an open subset of $\mathbb{R}^{2 n+1}$. Nondegeneracy is clearly an open condition among classical paths. A standard result holds that for given initial conditions, the set of durations for which a classical path fails to be nondegenerate is discrete; see e.g. [13]. In particular, the nondegenerate classical paths are a dense open subset of all classical paths. The following lemma follows from e.g. [9]; in Section 3.1 we include the proof rewritten in the language of classical paths.

LEMMA 2.1.4. Let $\gamma:[0, t] \rightarrow \mathbb{R}^{n}$ be classical and nondegenerate. Then there exists a neighborhood $\mathcal{O}$ of $(\gamma(0), \gamma(t)) \in \mathbb{R}^{2 n}$ and a map $\hat{\gamma}:[0, t] \times \mathcal{O} \rightarrow \mathbb{R}^{n}$ such that (i) $\hat{\gamma}\left(-, q_{0}, q_{1}\right)$ is classical and nondegenerate for each $\left(q_{0}, q_{1}\right) \in \mathcal{O}$, (ii) $\hat{\gamma}\left(0, q_{0}, q_{1}\right)=q_{0}$ and $\hat{\gamma}\left(t, q_{0}, q_{1}\right)=q_{1}$, and (iii) $\hat{\gamma}(-, \gamma(0), \gamma(t))=\gamma$.

Let $\gamma$ be a classical path of duration $t$. Then for sufficiently small $\epsilon, \gamma$ determines classical paths $\gamma_{s}$ for $s \in[t-\epsilon, t+\epsilon]$, either by restriction or by extension via equation (2.1.2), and if $\gamma$ is nondegenerate, for sufficiently small $\epsilon$ so are the $\gamma_{s}$. Thus, to a nondegenerate classical path $\gamma$ we can associate a family $\hat{\gamma}_{t}\left(q_{0}, q_{1},-\right)$ of nondegenerate classical paths, varying smoothly in the parameters. We will abuse notation and write $\gamma$ for this whole family. So henceforth $\gamma$ is a smooth family of solutions to the boundary value problem given by equation (2.1.2) along with Dirichlet boundary values $\gamma(0)=q_{0}, \gamma(t)=q_{1}$, where $\left(t, q_{0}, q_{1}\right)$ ranges over an open subset $\mathcal{O}$ of $\mathbb{R}^{2 n+1}$.

DEFINITION 2.1.5. Let $\gamma$ be a classical nondegenerate path, extended to a family with boundary values varying in $\mathcal{O} \subseteq \mathbb{R}^{2 n+1}$ as above. The corresponding Hamilton function $S_{\gamma}: \mathcal{O} \rightarrow \mathbb{R}$ is:

$$
S_{\gamma}\left(t, q_{0}, q_{1}\right)=\int_{0}^{t}\left(\frac{1}{2}|\dot{\gamma}(\tau)|^{2}+B_{i}(\gamma(\tau)) \dot{\gamma}^{i}(\tau)-C(\gamma(\tau))\right) \mathrm{d} \tau
$$


Then $S_{\gamma}\left(t, q_{0}, q_{1}\right)$ satisfies the following well-known equations:

$$
\begin{aligned}
& \frac{\partial\left[-S_{\gamma}\right]}{\partial t}=\frac{1}{2}\left|\nabla_{q_{0}}\left[-S_{\gamma}\right]-B\left(q_{0}\right)\right|^{2}+C\left(q_{0}\right)=\frac{1}{2}\left|\nabla_{q_{1}}\left[-S_{\gamma}\right]+B\left(q_{1}\right)\right|^{2}+C\left(q_{1}\right) \\
& \frac{\partial\left[-S_{\gamma}\right]}{\partial q_{0}^{i}}=\dot{\gamma}^{i}(0)+B_{i}\left(q_{0}\right) \quad \frac{\partial S_{\gamma}}{\partial q_{1}^{i}}=\dot{\gamma}^{i}(t)+B_{i}\left(q_{1}\right)
\end{aligned}
$$

For a few different constructions, we will need the matrix $\frac{\partial^{2}}{\partial q_{0} \partial q_{1}}\left[-S\left(t, q_{0}, q_{1}\right)\right]$ and its inverse; we define the indices on the inverse matrix by: $\left(\left(\frac{\partial^{2}[-S]}{\partial q_{1} \partial q_{0}}\right)^{-1}\right)^{k l}$ $\frac{\partial^{2}[-S]}{\partial q_{0}^{l} \partial q_{1}^{m}}=\delta_{m}^{k}$, and similarly for $q_{1} \leftrightarrow q_{0}$.

LEMMA 2.1.9. Let $\gamma$ be a nondegenerate path of duration $t$, extended to a family as above. Let $G_{\gamma}:[0, t]^{\times 2} \rightarrow\left(\mathbb{R}^{n}\right)^{\otimes 2}$ be defined by:

$$
\begin{aligned}
G_{\gamma}^{i j}(\varsigma, \tau)= & \Theta(\varsigma-\tau) \frac{\partial \gamma^{i}}{\partial q_{0}^{k}}(\varsigma)\left(\left(\frac{\partial^{2}\left(-S_{\gamma}\right)}{\partial q_{0} \partial q_{1}}\right)^{-1}\right)^{k l} \frac{\partial \gamma^{j}}{\partial q_{1}^{l}}(\tau) \\
& +\Theta(\tau-\varsigma) \frac{\partial \gamma^{i}}{\partial q_{1}^{k}}(\varsigma)\left(\left(\frac{\partial^{2}\left(-S_{\gamma}\right)}{\partial q_{1} \partial q_{0}}\right)^{-1}\right) \frac{\partial \gamma^{j}}{\partial q_{0}^{l}}(\tau)
\end{aligned}
$$

Then $-G_{\gamma}$ is a Green's function for the operator $\mathcal{D}_{\gamma}$ given in equation (2.1.3): i.e. $G_{\gamma}$ satisfies $\mathcal{D}_{\gamma}\left[G_{\gamma}\right]_{k}^{i}(\varsigma, \tau)=-\delta_{k}^{i} \delta(\tau-\varsigma)$, where $\mathcal{D}_{\gamma}$ acts on the $j, \tau$ parts of equation (2.1.10), and $G_{\gamma}$ vanishes along the boundary of the square $[0, t]^{\times 2}$.

For the proof, see Section 3.2. Statements similar to Lemmas 2.1.4 and 2.1.9 appear in e.g. [2], where degenerate paths are also briefly addressed.

\subsection{FEYNMAN DIAGRAMS AND THE MAIN RESULTS}

DEFINITION 2.2.1. A partition of a set $X$ is a collection of nonempty subsets of $X$, called the blocks of the partition, that are pairwise disjoint and whose union is all of $X$.

A Feynman diagram is a finite combinatorial graph with all vertices trivalent and higher. (Self-loops, parallel edges, and the empty diagram are allowed.) More precisely, a Feynman diagram is a finite set $H$ of "half-edges," along with two partitions $E$ (the "edges") and $V$ (the "vertices") of $H$, with the requirements $|e|=2$ for each block $e \in E$, and $|v| \geq 3$ for each block $v \in V$. An isomorphism of Feynman diagrams $\Gamma_{1}=\left(H_{1}, E_{1}, V_{1}\right)$ and $\Gamma_{2}=\left(H_{2}, E_{2}, V_{2}\right)$ is a bijection $\phi: H_{1} \stackrel{\sim}{\rightarrow}$ $H_{2}$ such that $\phi\left(E_{1}\right)=E_{2}$ and $\phi\left(V_{1}\right)=V_{2}$, where $\phi\left(E_{1}\right), \phi\left(V_{1}\right)$ are the obvious partitions of $H_{2}$ induced by $\left(\phi, E_{1}, V_{1}\right)$. We will denote Feynman diagrams with the 
obvious pictures:
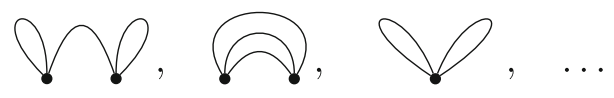

Let $\Gamma=(H, E, V)$ be a Feynman diagram. The Euler characteristic of $\Gamma$ is $\chi(\Gamma)=$ $|V|-|E|$. The connected components of $\Gamma$ are the equivalence classes of $H$ under the equivalence relation generated by $E \cup V$. Let $\pi_{0}(\Gamma)$ be the set of connected components of $\Gamma$; then $\lambda(\Gamma)=\left|\pi_{0}(\Gamma)\right|-\chi(\Gamma)$ is the number of loops of $\Gamma$, also called its first Betti number. A diagram $\Gamma$ is connected if it has precisely one connected component. For example, the above three pictures comprise all (up to isomorphism) diagrams $\Gamma$ with $\chi(\Gamma)=-1$, and each is connected.

A marked Feynman diagram is a Feynman diagram $\Gamma=(H, E, V)$ along with a subset $M \subseteq H$ of "marked half-edges" such at each vertex $v \in V$, at most one halfedge $\eta \in v$ is marked, i.e. $|v \cap M| \leq 1$. An isomorphism of marked Feynman diagrams is required to respect the marking. We indicate the markings on a marked Feynman diagram with small circles, as in equation (2.2.4).

DEFINITION 2.2.2. Pick a duration $t>0$. A totally labeled Feynman diagram (with duration $t)$ is a Feynman diagram $\Gamma=(H, E, V)$ along with maps $\vec{\tau}: V \rightarrow$ $[0, t]$ and $\vec{\jmath}: H \rightarrow\{1, \ldots, n\}$. Let $\gamma$ be a classical nondegenerate path of duration $t$ and $(\Gamma, M, \vec{\tau}, \vec{\jmath})$ a totally labeled marked Feynman diagram. Then its value is (no summation convention):

$$
\begin{aligned}
& \mathcal{F}_{\gamma}(\Gamma, M, \vec{\tau}, \vec{\jmath})=\prod_{v \in V, v \cap M=\emptyset}\left(\left.\left(\prod_{\eta \in V} \frac{\partial}{\partial q^{\vec{\jmath}(\eta)}}\right)[C(q)]\right|_{q=\gamma(\vec{\tau}(v))}\right. \\
& \left.+\left.\left(\prod_{\eta \in V} \frac{\partial}{\partial q^{\vec{\jmath}(\eta)}}\right)\left[B_{k}(q)\right]\right|_{q=\gamma(\vec{\tau}(v))} \dot{\gamma}^{k}(\tau)\right) \\
& \times \prod_{v \in V, v \cap M=\{\zeta\}}\left(\left.\left(\prod_{\eta \in v \backslash\{\zeta\}} \frac{\partial}{\partial q^{\vec{J}(\eta)}}\right)\left[-B_{\vec{J}(\zeta)}(q)\right]\right|_{q=\gamma(\vec{\tau}(v))}\right) \\
& \times \prod_{\substack{e=\{\eta, \zeta\} \in E, e \cap M=\emptyset}} G^{\vec{j}(\eta), \vec{j}(\zeta)}(\vec{\tau}(\eta), \vec{\tau}(\zeta)) \\
& \times\left.\prod_{\substack{e=\{\eta, \zeta\} \in E, e \cap M=\{\eta\}}} \frac{\partial G^{\vec{j}(\eta), \vec{j}(\zeta)}\left(\varsigma_{1}, \varsigma_{2}\right)}{\partial \varsigma_{1}}\right|_{\substack{\varsigma_{1}=\vec{\tau}(\eta) \\
\varsigma_{2}=\vec{\tau}(\zeta)}} \\
& \times\left.\prod_{\substack{e=\{\eta, \zeta\} \in E, e \subseteq M}} \frac{\partial^{2} G^{\vec{j}(\eta), \vec{\jmath}(\zeta)}\left(\varsigma_{1}, \varsigma_{2}\right)}{\partial \varsigma_{1} \partial \varsigma_{2}}\right|_{\substack{\varsigma_{1}=\vec{\tau}(\eta) \\
\varsigma_{2}=\vec{\tau}(\zeta)}}
\end{aligned}
$$


If $(\Gamma, M)$ is an (unlabeled) marked Feynman diagram, its value is an integral over all total labelings:

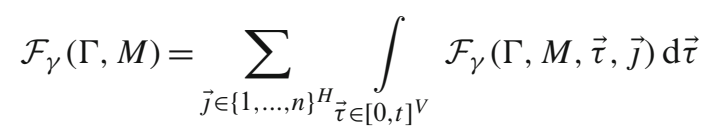

More compactly, Feynman diagrams are evaluated via the following Feynman rules and the obvious compositions and contractions (including the Einstein summation convention), where $\xi_{1}, \ldots, \xi_{k}$ are test-functions ranging over the space of paths of duration $t$ :

$$
\begin{aligned}
& \mathcal{F}_{\gamma}(\underbrace{\xi_{1} \xi_{2}} \cdots \xi^{\xi_{n}})=\left.\int_{\tau=0}^{t}\left(\frac{\partial^{n} C(q)}{\partial q^{i_{1}} \ldots \partial q^{i_{n}}}+\frac{\partial^{n} B_{k}(q)}{\partial q^{i_{1}} \cdots \partial q^{i_{n}}} \dot{\gamma}^{k}(\tau)\right)\right|_{q=\gamma(\tau)} \xi_{1}^{i_{1}}(\tau) \cdots \xi_{n}^{i_{n}}(\tau) d \tau \\
& \mathcal{F}_{\gamma}(\underbrace{\xi_{1} \xi_{2}} \underbrace{\xi_{n}})=\left.\int_{\tau=0}^{t} \frac{\partial^{n-1}\left[-B_{i_{1}}\right]}{\partial q^{i_{2}} \cdots \partial q^{i_{n}}}\right|_{q=\gamma(\tau)} \dot{\xi}_{1}^{i_{1}}(\tau) \xi_{2}^{i_{2}}(\tau) \cdots \xi_{n}^{i_{n}}(\tau) d \tau \\
& \mathcal{F}_{\gamma}\left(\bigcap_{\tau, i}\right)=G_{\gamma}^{i j}(\varsigma, \tau)
\end{aligned}
$$

We must check that the value of a marked Feynman diagram converges: the Green's function $G_{\gamma}(\zeta, \tau)$ is non-smooth like $|\varsigma-\tau|$ near the diagonal, so any diagram in which both ends of the same edge are marked includes a Dirac $\delta$-function distribution. But no vertex can have more than one marking, so such $\delta$-functions do not lead to divergence integrals. (We have insisted that $B, C$ be smooth. If they are allowed to be singular, divergences can occur, requiring some renormalization procedure, c.f. [12].) We prove the following in Section 4.

THEOREM 2.2.6 (Schrödinger's Equation). Let $\gamma$ be a classical nondegenerate path, extended to a family with boundary values $\left(t, q_{0}, q_{1}\right)$ ranging over some open set $\mathcal{O} \in \mathbb{R}^{2 n+1}$. Define on $\mathcal{O}$ the following $\mathbb{R}[[i \hbar]]$-valued function:

$$
\begin{aligned}
V_{\gamma}\left(t, q_{0}, q_{1}\right)= & -S_{\gamma}\left(t, q_{0}, q_{1}\right)+i \hbar \frac{1}{2} \log \left|\operatorname{det} \frac{\partial^{2}\left[-S_{\gamma}\right]}{\partial q_{0} \partial q_{1}}\right| \\
& +\sum_{\begin{array}{c}
\text { equivalence classes }(\Gamma, M) \\
\text { of connected marked } \\
\text { Feynman diagrams }
\end{array}} \frac{(i \hbar)^{\lambda(\Gamma)} \mathcal{F}_{\gamma}(\Gamma, M)}{|\operatorname{Aut}(\Gamma, M)|}
\end{aligned}
$$

Then $U_{\gamma}=\exp \left((i \hbar)^{-1} V_{\gamma}\right)$ is a formal solution to Schrödinger's equation: $i \hbar \frac{\partial U_{\gamma}}{\partial t}=$ $\hat{H}\left[U_{\gamma}\right]$ as formal power series in $\hbar$.

We remark that for a connected diagram $\Gamma=(H, E, V)$, we have $\lambda(\Gamma)=|E|-$ $|V|+1 \leq|E|=2|H|$, and so the sum in equation (2.2.7) is finite at each order in $\hbar$. 
In general, the series has zero radius of convergence in $\hbar$. Note that $U_{\gamma}$ can be described as:

$$
U_{\gamma}\left(t, q_{0}, q_{1}\right)=e^{-(i \hbar)^{-1}} S_{\gamma} \sqrt{\left|\operatorname{det} \frac{\partial\left[-S_{\gamma}\right]}{\partial q_{0} \partial q_{1}}\right|} \sum_{\begin{array}{c}
\text { equivalence classes of } \\
\text { marked Feynman } \\
\text { diagrams }(\Gamma, M)
\end{array}} \frac{(i \hbar)^{-\chi(\Gamma)} \mathcal{F}_{\gamma}(\Gamma, M)}{|\operatorname{Aut}(\Gamma, M)|}
$$

\subsection{SUMS AND LIMITS OF $U_{\gamma}$}

Temporarily, let $\hbar$ range over "small" non-zero real numbers. Pick $N$ a positive integer and suppose that $\mathcal{O} \subseteq \mathbb{R}^{n}$ is open, and $f_{1}, f_{2}: \mathcal{O} \rightarrow \mathbb{R}[i \hbar]$ satisfy $f_{1}=f_{2}+$ $O\left(\hbar^{N+1}\right)$. Let $g: \mathcal{O} \rightarrow \mathbb{R}^{n}$ have compact support. Then:

$$
\int e^{(i \hbar)^{-1} f_{1}(q)} g(q) \mathrm{d} q=\int e^{(i \hbar)^{-1} f_{2}(q)} g(q) \mathrm{d} q+O\left(\hbar^{N+n / 2}\right)
$$

For details, see [4, Chapter 3]. Thus, if $f, g: \mathcal{O} \rightarrow \mathbb{R}[[i \hbar]]$ are smooth term-by-term and $g$ has compact support, then we are justified in defining $\int \exp \left((i \hbar)^{-1} f(q)\right)$ $g(q) \mathrm{d} q$ as a formal expression in $\hbar$, by successively approximating $f$ by polynomials in $\hbar$. We will write $\int^{\text {formal }}$ for this formal integral.

We will not totally define what we mean by a "formal expression in $\hbar$," although it is not hard to do so. Essentially, a formal expression in $\hbar$ is a $\mathbb{C}[[\sqrt{\hbar}]]$-linear combination of terms of the form $\exp \left((i \hbar)^{-1} f\right)$, where $f \in \mathbb{R}[[i \hbar]]$, modulo the obvious equivalences and with the obvious arithmetic. If $f(\bmod \hbar)$ has finitely many critical points within the support of $g$, and if at each critical point the Hessian of $f$ is nondegenerate, then $\int^{\text {formal }} \exp \left((i \hbar)^{-1} f\right) g$ is a formal expression in $\hbar$ in this sense.

DEFINITION 2.3.2. Let $f_{1}, f_{2}, \ldots, f_{\infty}$ be distributions on $\mathcal{O} \subseteq \mathbb{R}^{n}$, valued in formal expressions in $\hbar$. Then $\lim f_{N}=f_{\infty}$ if and only if for every $g: \mathcal{O} \rightarrow \mathbb{R}$ with compact support, $\lim _{N \rightarrow \infty} \int_{\mathcal{O}}^{\text {formal }} f_{N}(q) g(q) \mathrm{d} q=\int_{\mathcal{O}}^{\text {formal }} f_{\infty}(x) g(x) \mathrm{d} x$. This defines the pointwise convergence topology on the space of distributions. The topology on $\mathbb{R}[[i \hbar]]$ is given coefficient-by-coefficient.

In Section 5 we will prove:

THEOREM 2.3.3 (The initial value problem). Pick $\mathcal{O} \subseteq \mathbb{R}^{2 n+1}$ open, and let $A$ be the set of all families of nondegenerate classical paths with boundary values varying over $\mathcal{O}$. Let $\left\{g_{\gamma}\right\}_{\gamma \in A}$ be a collection of smooth functions $\mathcal{O} \rightarrow \mathbb{R}[[i \hbar]]$. Then for fixed $t, q_{1}$, the possibly-infinite sum $\sum_{\gamma \in A} g_{\gamma}\left(t, q_{0}, q_{1}\right) \exp \left((i \hbar)^{-1} V_{\gamma}\left(t, q_{0}, q_{1}\right)\right)$ converges pointwise as a distribution in $q_{0}$ valued in formal expressions in $\hbar$.

Moreover, suppose that $\mathcal{O}=(0, \epsilon) \times \mathcal{O}_{0} \times \mathcal{O}_{0}$, where $\mathcal{O}_{0} \subseteq \mathbb{R}^{n}$ is open with compact closure and $\epsilon>0$ is sufficiently small depending on $\mathcal{O}_{0}$. Then for fixed $q_{1}$, as a 
distribution valued in formal expressions in $\hbar$ we have:

$$
\lim _{t \rightarrow 0} \sum_{\gamma \in A} \exp \left((i \hbar)^{-1} V_{\gamma}\left(t, q_{0}, q_{1}\right)\right)=(2 \pi \hbar)^{n / 2} e^{i n \pi / 4} \delta\left(q_{0}-q_{1}\right)
$$

The reader should be mildly disappointed in Theorem 2.3.3: we have first set $\hbar$ to a formal parameter, and then, in equation (2.3.4), taken a limit as $t \rightarrow 0$. Much better would be to approximate $V_{\gamma}$ by a polynomial in $\hbar$, interpret $\hbar$ as a positive real parameter, take any necessary limits, and then take the asymptotics as $\hbar \rightarrow 0$. But when the limits are performed in this order, we do not believe that $\sum_{\gamma \in A} \exp \left((i \hbar)^{-1} V_{\gamma}\right)$ always converges. Moreover, even if it does, some extra assumption is needed in order to take the $t \rightarrow 0$ limit for non-formal $\hbar$ in equation (2.3.4). For example, it is enough if the paths $\gamma \in A$ are of bounded length, although we will not prove this. However, in as natural a system as the onedimensional mechanics with electric potential $C(q)=q^{4}$, the classical paths can be arbitrarily long, and as $t \rightarrow 0$ with fixed endpoints the classical paths run away with length growing as $t^{-1 / 2}$.

One final remark is in order. It is tempting to conclude from Theorems 2.2.6 and 2.3.3 that the $\hbar \rightarrow 0$ asymptotics of the full propagator $U\left(t, q_{0}, q_{1}\right)$ are be given by:

$$
U\left(t, q_{0}, q_{1}\right) \approx \sum_{\substack{\gamma \text { classical, } \\ \gamma(0)=q_{0}, \gamma(t)=q_{1}}}(2 \pi \hbar)^{-n / 2} e^{-i n \pi / 4} \exp \left((i \hbar)^{-1} V_{\gamma}\left(t, q_{0}, q_{1}\right)\right)
$$

But a modification must be made. The formal power series $V_{\gamma}$ are defined only for $\gamma$ nondegenerate, and Theorem 2.2.6 only applies in this case. Thus the above formal sum solves equation (1.0.2) only for short times in a neighborhood of the diagonal. In general, if we know the asymptotics of $U$ near short nondegenerate paths, we can determine the full asymptotics by means of the composition law: $U\left(t_{0}+t_{1}, q_{0}, q_{1}\right)=\int U\left(t_{0}, q_{0}, q\right) U\left(t_{1}, q, q_{1}\right) \mathrm{d} q$. In [8] we prove the following:

THEOREM 2.3.5 (Semigroup law). Let $\gamma, V_{\gamma}$ be as in Theorem 2.2.6. Suppose that $t=t_{0}+t_{1}$ and the restrictions $\gamma_{0}=\left.\gamma\right|_{\left[0, t_{0}\right]}$ and $\gamma_{1}=\left.\gamma\right|_{\left[t_{0}, t\right]}$ are nondegenerate, and define the corresponding formal power series $V_{\gamma_{0}}$ and $V_{\gamma_{1}}$. Let $\eta(\gamma)$ be the Morse index of $\gamma$ with respect to the Morse function $\gamma \mapsto \int_{0}^{t}\left(\frac{1}{2}|\dot{\gamma}|^{2}+B(\gamma) \cdot \dot{\gamma}-C(\gamma)\right) \mathrm{d} \tau$ on the space of paths with the same boundary values, and similarly define Morse indexes $\eta\left(\gamma_{0}\right)$ and $\eta\left(\gamma_{1}\right)$ (see e.g. [13]). Set $U_{\gamma}=(-i)^{\eta(\gamma)}(2 \pi \hbar)^{-n / 2} e^{-i n \pi / 4} \exp \left((i \hbar)^{-1}\right.$ $\left.V_{\gamma}\left(t, q_{0}, q_{1}\right)\right)$, and similarly define $U_{\gamma_{0}}$ and $U_{\gamma_{1}}$. Then for a sufficiently small neighborhood $\mathcal{Q}$ of $\gamma\left(t_{0}\right)$ we have:

$$
U_{\gamma}\left(t_{0}+t_{1}, q_{0}, q_{1}\right)=\int_{\mathcal{Q}}^{\text {formal }} U_{\gamma_{0}}\left(t_{0}, q_{0}, q\right) U_{\gamma_{1}}\left(t_{1}, q, q_{1}\right) \mathrm{d} q
$$


Thus, the correct asymptotics of the full propagator $U\left(t, q_{0}, q_{1}\right)$ are essentially given by:

$$
U\left(t, q_{0}, q_{1}\right) \approx(2 \pi \hbar)^{-n / 2} e^{i n \pi / 4} \sum_{\substack{\gamma \text { classical, } \\ \gamma(0)=q_{0}, \gamma(t)=q_{1}}}(-i)^{\eta(\gamma)} \exp \left((i \hbar)^{-1} V_{\gamma}\left(t, q_{0}, q_{1}\right)\right)
$$

Here the sum makes sense provided that there is no degenerate classical path of duration $t$ connecting $q_{0}$ to $q_{1}$, and converges pointwise as a distribution. This justifies the method of formal Feynman path integration.

\section{Proofs of the Lemmas}

\subsection{PROOF OF LEMMA 2.1.4}

Let $\mathcal{P}$ be the space of paths of duration $t$ and $\Lambda$ the space of based loops of duration $t$. Each is an infinite-dimensional vector space; we think of $\mathcal{P}$ as an infinitedimensional smooth manifold with tangent bundle $\mathrm{T} \mathcal{P}=\mathcal{P} \times \mathcal{P}$. Recall that the derivative $d f$ of a function $f: \mathcal{P} \rightarrow \mathbb{R}$ is defined by $d f_{\gamma} \cdot \xi=\left.\frac{\partial}{\partial \epsilon}\right|_{\epsilon=0}(\gamma+\epsilon \xi) ; f$ is differentiable if such a derivative exists for all $(\xi, \gamma) \in \mathrm{T} \mathcal{P}$. Let $\pi: \mathcal{P} \rightarrow \mathbb{R}^{2 n}$ be the map $\gamma \mapsto(\gamma(0), \gamma(t))$; it is a trivial bundle with fiber $\Lambda$. As $\Lambda$ is a vector space, we can identify the fiber tangent spaces: if $\gamma \in \mathcal{P}$, then $\mathrm{T}_{\gamma}\left(\pi^{-1}(\pi(\gamma))\right)=\Lambda$.

Consider the function $f: \mathcal{P} \rightarrow \mathbb{R}$ given by:

$$
f(\gamma)=\int_{0}^{t}\left(\frac{1}{2}|\dot{\gamma}(\tau)|^{2}+B_{i}(\gamma(\tau)) \dot{\gamma}^{i}(\tau)-C(\gamma(\tau))\right) \mathrm{d} \tau
$$

As is well-known, a path $\gamma$ is classical if and only if $\Lambda \subseteq \operatorname{ker} d f_{\gamma}$. The Hessian of $f$ at $\gamma$ is the symmetric bilinear form:

$$
\begin{aligned}
h_{\gamma}:\left(\xi_{1}, \xi_{2}\right) \mapsto & \int_{0}^{t}\left(\dot{\xi}_{1}^{i}(\tau) \dot{\xi}_{2}^{j}(\tau)+\frac{\partial B_{i}(q)}{\partial q^{j}}\left(\dot{\xi}_{1}^{i}(\tau) \xi_{2}^{j}(\tau)+\dot{\xi}_{1}^{j}(\tau) \xi_{2}^{i}(\tau)\right)\right. \\
& \left.+\left(\frac{\partial^{2} B_{k}(q)}{\partial q^{i} \partial q^{j}} \dot{\gamma}^{k}(\tau)-\frac{\partial^{2} C(q)}{\partial q^{i} \partial q^{j}}\right) \xi_{1}^{i}(\tau) \xi_{2}^{j}(\tau)\right)\left.\right|_{q=\gamma(\tau)} \mathrm{d} \tau
\end{aligned}
$$

When $\xi_{1}, \xi_{2}$ are based loops, integration by parts gives $h_{\gamma}\left(\xi_{1}, \xi_{2}\right)=\int_{0}^{t} \xi_{1}(\tau)$. $\mathcal{D}_{\gamma}\left[\xi_{2}\right](\tau) \mathrm{d} \tau$. Thus $\gamma$ is nondegenerate if and only if $\Lambda \cap \operatorname{ker} h_{\gamma}=0$.

Let $\mathcal{C}$ be the space of classical paths. By considering the derivatives of $d f$ along paths in $\mathcal{C}$, one easily checks that $\mathrm{T}_{\gamma} \mathcal{C} \subseteq \operatorname{ker} h_{\gamma}$. Thus if $\gamma$ is classical and nondegenerate, then $d \pi: \mathrm{T}_{\gamma} \mathcal{C} \rightarrow \mathrm{T}_{\pi(\gamma)} \mathbb{R}^{2 n}$ is an injection. But the space of classical paths of a given duration has dimension $2 n$, and so $d \pi$ is a bijection. This completes the proof of Lemma 2.1.4. 


\subsection{PROOF OF LEMMA 2.1.9}

We begin with the observation that for each $a=0,1$ and each $j=\{1, \ldots, n\}$, the path $\phi_{a, j}(\tau)=\frac{\partial \gamma}{\partial q_{a}^{j}}(\tau)$ satisfies $\mathcal{D}_{\gamma}[\phi]=0$; this follows by differentiating equation (2.1.2). In other words, the $2 n$ paths $\phi_{a, j}$ are Jacobi fields along $\gamma$. The boundary values are: $\phi_{0, j}^{i}(0)=\delta_{j}^{i}=\phi_{1, j}^{i}(t)$ and $\phi_{0, j}^{i}(t)=\delta_{j}^{i}=\phi_{1, j}^{i}(0)$. Let $\phi_{0}(\tau)$, $\phi_{1}(\tau)$ be the $n \times n$ matrices $\left(\phi_{a}(\tau)\right)_{j}^{i}=\phi_{a, j}^{i}(\tau)$. Each solution to the initial value problem $\left\{\mathcal{D}_{\gamma}[\varphi], \varphi(0)=0\right\}$ is determined by $\dot{\varphi}(0)$. Since $\phi_{1}(t)=\delta$ is full-rank, $\dot{\phi}_{1}(0)$ must be also.

Define the following function on $[0, t]$ valued in $2 n \times 2 n$ matrices:

$$
M(\tau)=\left(\begin{array}{ll}
\phi_{0}(\tau) & \phi_{1}(\tau) \\
\dot{\phi}_{0}(\tau) & \dot{\phi}_{1}(\tau)
\end{array}\right)
$$

Then $M(0)$ is lower-triangular with full-rank blocks on the diagonal, and hence invertible. Since the coefficient on $\dot{\xi}$ in $\mathcal{D}_{\gamma}[\xi]$ is traceless, Liouville's formula gives $\operatorname{det} M(\tau)=\operatorname{det} M(0)$, and so $M(\tau)$ is invertible for each $\tau \in[0, t]$. Let $\psi_{0}, \psi_{1}$ be the $(n \times n)$-matrix valued functions on $[0, t]$ satisfying:

$$
\left(\begin{array}{ll}
\phi_{0, j}^{i}(\tau) & \phi_{1, j}^{i}(\tau) \\
\dot{\phi}_{0, j}^{i}(\tau) & \dot{\phi}_{1, j}^{i}(\tau)
\end{array}\right)\left(\begin{array}{c}
\psi_{0, k}^{j}(\tau) \\
\psi_{1, k}^{j}(\tau)
\end{array}\right)=\left(\begin{array}{c}
0 \\
\delta_{k}^{i}
\end{array}\right)
$$

Then it is straightforward to check that the Green's function for $-\mathcal{D}_{\gamma}$ is given by:

$$
G_{k}^{i}(\varsigma, \tau)=-\Theta(\varsigma-\tau) \phi_{0, j}^{i}(\tau) \psi_{0, k}^{j}(\varsigma)+\Theta(\tau-\varsigma) \phi_{j}^{1, i}(\tau) \psi_{1, k}^{j}(\varsigma)
$$

But the differential operator $-\mathcal{D}_{\gamma}$ is self-adjoint for the inner product $\left\langle\xi_{1}, \xi_{2}\right\rangle=$ $\int_{0}^{t} \xi_{1} \cdot \xi_{2}$ on the space of based loops. Thus if $G_{k}^{i}(\varsigma, \tau)$ is its Green's function, then $G_{k}^{i}(\varsigma, \tau)=G_{i}^{k}(\tau, \varsigma)$. In particular, each $\psi_{a}$ satisfies $\mathcal{D}_{\gamma}\left[\psi_{a}\right]=0$, and so the $\psi_{a}$ s are linear combinations of the $\phi_{b}$ s. To find the coefficients, we need only check the boundary values. We have $\psi_{0, k}^{j}(0)=0=\psi_{1, k}^{j}(t)$, by evaluating the top row of equation (3.2.1) at $\tau=0, t$. On the other hand, the bottom row then gives:

$$
\left(\psi_{0}(t)\right)^{-1}=\dot{\phi}_{0}(t)=\left.\frac{\partial^{2} \gamma(\tau)}{\partial \tau \partial q_{0}}\right|_{\tau=t}=\frac{\partial}{\partial q_{0}}(\dot{\gamma}(t))=\frac{\partial}{\partial q_{0}}\left(\frac{\partial S_{\gamma}}{\partial q_{1}}-B\left(q_{1}\right)\right)=\frac{\partial^{2} S_{\gamma}}{\partial q_{0} \partial q_{1}}
$$

We have used equation (2.1.8) to do the necessary simplifications. Thus $\psi_{0, k}^{j}(\varsigma)=\phi_{1, l}^{j}(\varsigma)\left(\left(\frac{\partial^{2} s_{\gamma}}{\partial q_{0} \partial q_{1}}\right)^{-1}\right)^{l k}$. A similar argument shows that $\psi_{1, k}^{j}(\varsigma)=$ $-\phi_{0, l}^{j}(\varsigma)\left(\left(\frac{\partial^{2} S_{\gamma}}{\partial q_{1} \partial q_{0}}\right)^{-1}\right)^{l k}$. This completes the proof of Lemma 2.1.9. 


\section{Proof of the Main Theorem}

The Schrödinger equation for $\exp \left((i \hbar)^{-1} V_{\gamma}\right)$ is equivalent to the following nonlinear differential equation for $V_{\gamma}$ :

$$
\frac{\partial V_{\gamma}}{\partial t}=\frac{1}{2}\left|\nabla_{q_{1}} V_{\gamma}\right|^{2}+\frac{i \hbar}{2} \Delta_{q_{1}} V_{\gamma}+B_{j}\left(q_{1}\right) \frac{\partial V_{\gamma}}{\partial q_{1}^{j}}+\frac{i \hbar}{2} \frac{\partial B_{j}\left(q_{1}\right)}{\partial q_{1}^{j}}+\left(\frac{1}{2}\left|B\left(q_{1}\right)\right|^{2}+C\left(q_{1}\right)\right)
$$

Our strategy will be to convert equation (4.0.1) into the diagrammatic language introduced earlier, whence it will follow from a few calculations of derivatives and some simple combinatorics.

\subsection{MORE FEYNMAN RULES}

In addition to the Feynman rules given in equations (2.2.4) to (2.2.5), we introduce the following notation to our graphical calculus:

$$
\mathcal{F}_{\gamma}\left(\begin{array}{cc}
, & - \\
i & j \\
i & j
\end{array}\right)=\delta^{i j} \quad \mathcal{F}_{\gamma}(C)=C\left(q_{1}\right) \quad \mathcal{F}_{\gamma}(B)=B_{i}\left(q_{1}\right) \quad \mathcal{F}_{\gamma}\left(\begin{array}{c}
\gamma \\
j
\end{array}\right)=\gamma^{i}(\tau)
$$

In particular, dashed lines carry only an index (no time variable). Contraction of indices is implied by connecting dashed strands.

We will henceforth largely drop the " $\mathcal{F}_{\gamma}$ " notation from equations of diagrams. We will find it convenient to abbreviate the two kinds of vertices in marked Feynman diagrams:

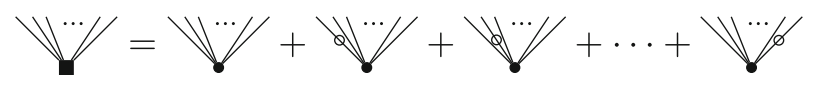

Then rather than discussing marked Feynman diagrams, we will simply say "Feynman diagram" and mean a diagram drawn with m-type vertices. It is clear that the automorphism counts work out: if adding some markings to a diagram divides the size of the autmorphism group by some number $n$, then there were $n$ equivalent ways to add those markings to the unmarked diagram. In particular a symmetry of a diagram with a -type vertex either acts as a symmetry on each of the expanded diagrams or permutes the possible expansions, and those that it permutes have their symmetry groups broken by the right amount.

We will also extend the possible valence of such vertices in our notation, although not in the sums of diagrams:

$$
\begin{aligned}
& \mathbf{\square}=-S_{\gamma} \\
& \xi
\end{aligned}
$$


Here the test functions $\xi, \zeta$ are paths of duration $t$, and in the second line on the right-hand side the bivalent vertices have the natural meanings extending equations (2.2.3) and (2.2.4) to $n=2$. In particular, $\stackrel{\xi}{=}=0$ if $\xi$ is a based loop. By integrating by parts, $\mho^{\xi}=\int_{0}^{t} \xi(\tau) \cdot \mathcal{D}_{\gamma}[\zeta](\tau) \mathrm{d} \tau$ whenever $\xi$ is a based loop, where $\mathcal{D}_{\gamma}$ is the differential operator given in equation (2.1.3).

We represent differentiation with respect to $t, q_{0}, q_{1}$ with dotted circles:
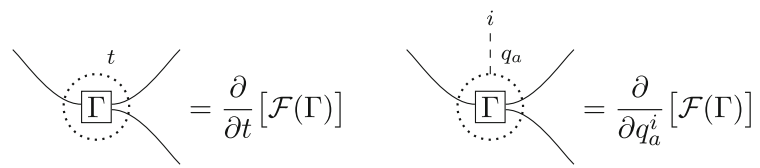

Then the product rule can be written graphically as:

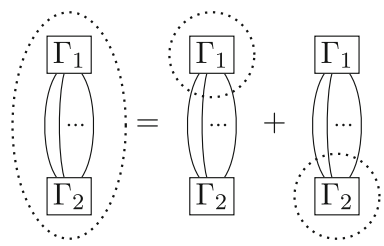

Suppose then that $\Gamma_{1}$ is a subdiagram of $\Gamma$ whose images $\Gamma_{1}, \ldots, \Gamma_{n}$ under the group of automorphisms of $\Gamma$ do not intersect, so that $\Gamma=\bar{\Gamma} \cup \Gamma_{1} \cup \cdots \cup \Gamma_{n}$ (we do allow automorphism of $\Gamma$ to induce nontrivial automorphisms of $\Gamma_{1}$ ). Then:

$$
\begin{aligned}
\Gamma & =\bar{\Gamma} \Gamma_{1} \Gamma_{2} \cdots \Gamma_{n} \cdots=\bar{\Gamma} \Gamma_{1} \Gamma_{2} \cdots \Gamma_{n}+\bar{\Gamma} \Gamma_{1} \Gamma_{2} \cdots \Gamma_{n}+\cdots+\bar{\Gamma} \Gamma_{1} \Gamma_{2} \cdots \Gamma_{n} \\
& =\bar{\Gamma} \Gamma_{1} \Gamma_{2} \cdots \Gamma_{n}+n \bar{\Gamma} \Gamma_{1} \Gamma_{2} \cdots \Gamma_{n}
\end{aligned}
$$

It is an elementary counting lemma that $\left|\operatorname{Aut}\left(\bar{\Gamma} \Gamma_{1} \Gamma_{2} \cdots \Gamma_{n}\right)\right|=\mid \frac{1}{n} \operatorname{Aut}\left(\bar{\Gamma} \Gamma_{1} \Gamma_{2} \cdots\right.$ $\left.\Gamma_{n}\right) \mid$. From this observation, we derive the fundamental result that for $z=q_{1}^{i}$ or $t$ :

$$
\begin{aligned}
& \frac{\partial}{\partial z} \sum_{\Gamma \text { connected }} \frac{(i \hbar)^{\lambda(\Gamma)} \mathcal{F}_{\gamma}(\Gamma)}{\mid \text { Aut } \Gamma \mid}=\sum_{\Gamma \text { connected with }} \frac{(i \hbar)^{\lambda(\Gamma)} \mathcal{F}_{\gamma}(\Gamma)}{\mid \text { Aut } \Gamma \mid} \\
& \text { one }
\end{aligned}
$$

The sum on the right-hand side ranges over connected components with precisely one differentiated basic subgraph - either a single differentiated vertex (of valence three or more) or a single differentiated edge.

In this notation, equation (2.1.7) reads:

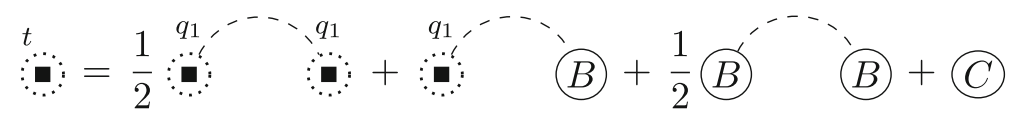


Moreover, it's clear that $\frac{\partial \gamma(\tau)}{\partial t}=-\frac{\partial \gamma(\tau)}{\partial q_{1}^{i}} \dot{\gamma}^{i}(t)$. Given equation (2.1.8), we have:

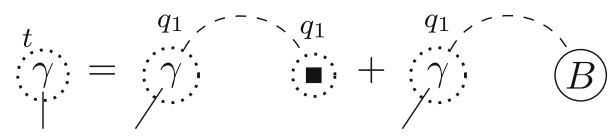

Finally, we introduce the Feynman rule ${ }^{0\left(\partial^{2}[-S]\right)^{-1}}$ for the inverse matrix $\left(\frac{\partial^{2}[-S]}{\partial q_{0} \partial q_{1}}\right)^{-1}$ :

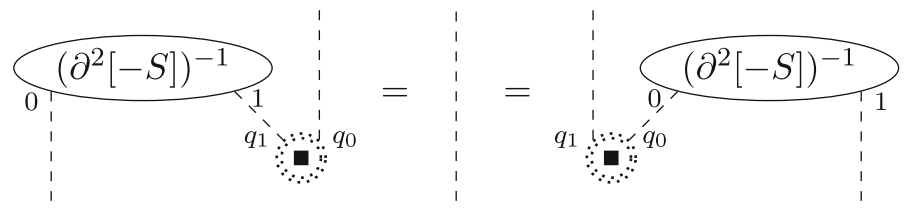

For example, in this notation equation (2.1.10) reads:

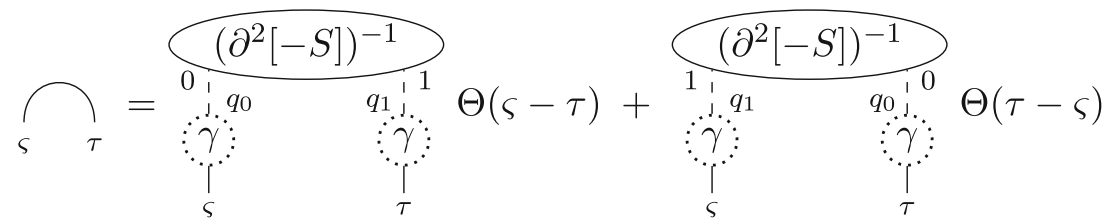

\subsection{DERIVATIVES OF THE VERTICES}

In this section we describe the derivatives of the vertices. We begin with the derivatives with respect to $q_{a}$. Equation (2.1.8) implies:

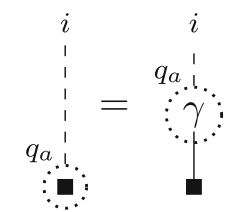

Integration by parts and the observations in the first paragraph of Section 3.2 give:

$$
j_{0}^{\xi}=\int_{0}^{t} \mathcal{D}_{\gamma}\left[\frac{\partial \gamma(\tau)}{\partial q_{a}}\right] \cdot \xi(\tau) d \tau+\left.\left(-\frac{\partial \dot{\gamma}^{j}(\tau)}{\partial q_{a}^{i}} \xi^{j}(\tau)-\frac{\partial B_{k}}{\partial q^{j}} \frac{\partial \gamma^{k}(\tau)}{\partial q_{a}^{i}} \xi^{j}(\tau)\right)\right|_{\tau=0} ^{t}=j^{\xi}
$$

In valence $n \geq 2$, the derivatives are particularly easy. Differentiating under the integral sign and using the chain rule gives:

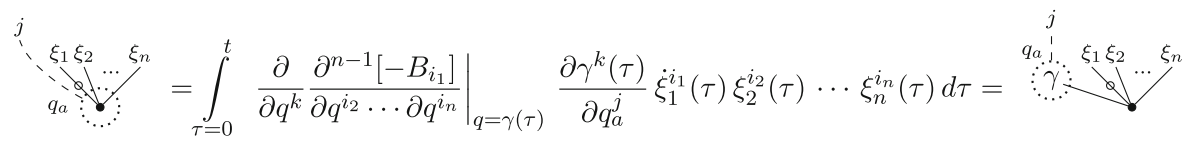


A similar argument gives:

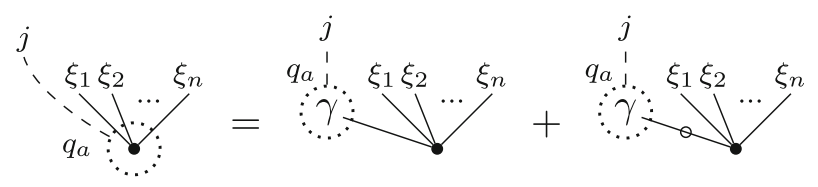

Summing and noting also that the non-diagrammatic part of the bivalent vertex does not depend on the $q_{a}$ s, we have, for all $n$ :

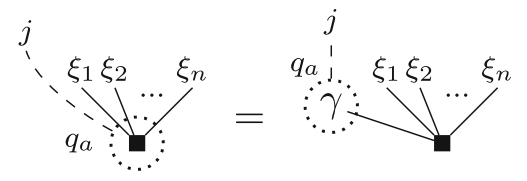

The derivatives with respect to $t$ are harder because of the boundary terms. At valence $n=0$, equation (4.1.2) already describes ${ }^{t}$. We will not describe the other $t$-derivatives in full generality, as we will only need them for valence $n \geq 3$ when all incoming edges are based loops. In this case, when $n \geq 3$ at least one incoming edge is not marked, and hence the boundary terms vanish. We have:

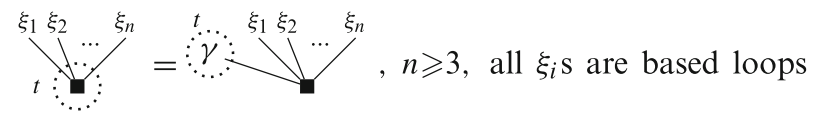

We conclude this section by addressing the second derivative of a vertex with respect to the $q_{a}$. By the product rule:

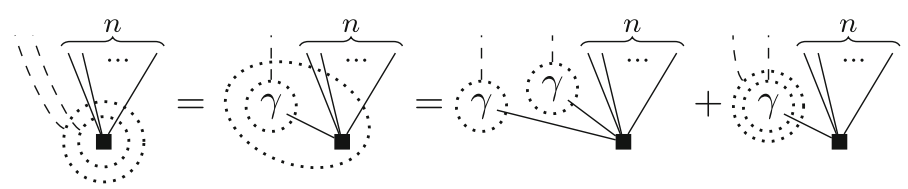

In particular:

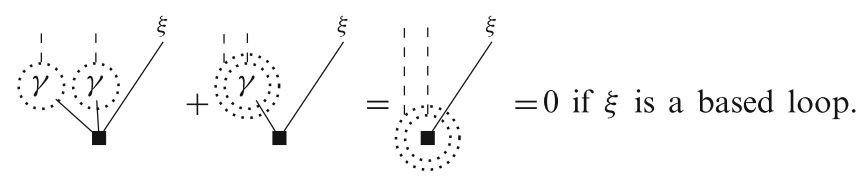

The Green's function $G_{\gamma}$, which we denote by an edge, is a based loop in each variable, so:

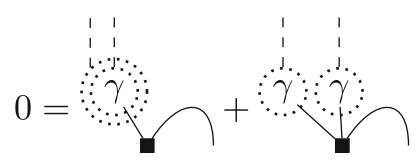


We have observed already that when contracted with based loops, the bivalent vertex acts as $\mathcal{D}_{\gamma}$. But $\mathcal{D}_{\gamma}\left[G_{\gamma}\right]_{j}^{i}(\varsigma, \tau)=-\delta_{j}^{i} \delta(\varsigma-\tau)$ by definition. In short-hand:

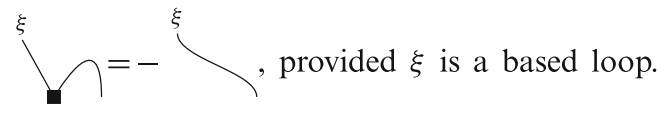

And $\frac{\partial^{2} \gamma(\tau)}{\partial q_{a} \partial q_{b}}$ vanishes at both endpoints $\tau=0, t$. Therefore:

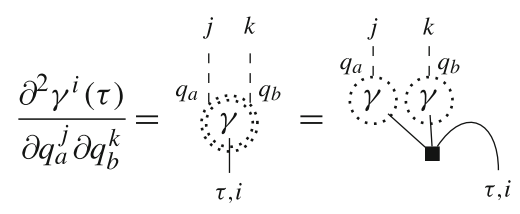

Thus in general to take the second derivative of a vertex one either adds two $\frac{\partial \gamma}{\partial q_{a}} \mathrm{~s}$ or an edge connecting to a trivalent vertex with two $\frac{\partial \gamma}{\partial q_{a}} \mathrm{~s}$. The lowest-valence example is:

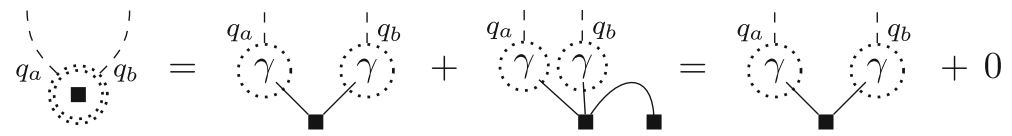

The second summand vanishes because $G_{\gamma}$ is a based loop in each variable. As we will need it later, we record one further derivative:

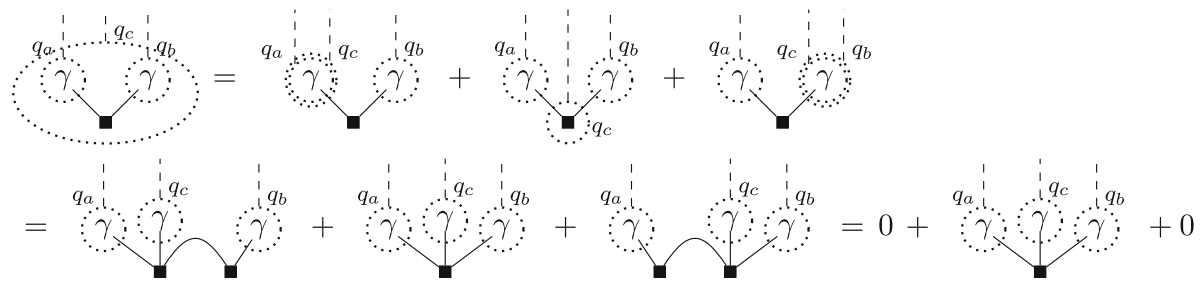

\subsection{DERIVATIVES OF THE GREEN'S FUNCTION AND THE DETERMINANT}

In this section, we evaluate the derivatives of the Green's function $G_{\gamma}$ given by equation (2.1.10) with respect to $q_{a}, t$. We record also the derivatives of $\log \left|\operatorname{det} \frac{\partial^{2}\left[-S_{\gamma}\right]}{\partial q_{0} \partial q_{1}}\right|$.

It is easy to evaluate $\frac{\partial G_{\gamma}}{\partial q_{a}}$. Let $\xi$ be a based loop, and consider the derivative of equation (4.2.3):

$$
0=\bigcap_{\vdots}^{\xi}=\varkappa^{\xi}+\overbrace{}^{\xi}
$$


We contract with $G_{\gamma}$, which is a based loop in each variable, and use equations (4.2.1) and (4.2.3):

$$
\because q_{a}=-\because q_{a}=
$$

The first equality in equation (4.3.2) requires that $\frac{\partial G_{\gamma}}{\partial q_{a}}$ is a based loop, which follows from differentiating $G_{\gamma}(\varsigma, 0)=0=G_{\gamma}(\varsigma, t)$ with respect to $q_{a}$.

Evaluating $\frac{\partial G}{\partial t}$ is not so easy, because it is not a based loop: from equation (4.3.9) it will follow that $\frac{\partial G^{i j}}{\partial t}(t, t)=\delta^{i j}$. Instead, we will differentiate equation (4.1.4) directly. To begin, we evaluate $\frac{\partial^{2} \gamma}{\partial t \partial q_{a}}$ by differentiating equation (4.1.3) with respect to $q_{a}$, using equations (4.2.4) and (4.2.5):

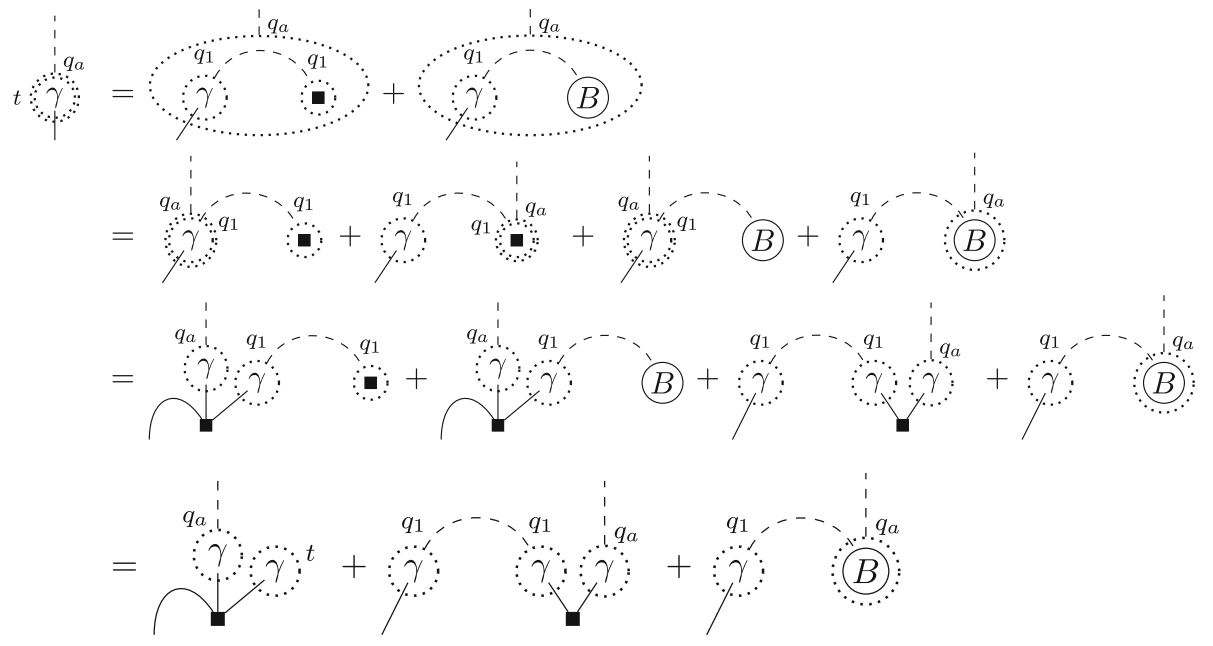

We remark that $(B)^{q_{0}}=\frac{\partial B\left(q_{1}\right)}{\partial q_{0}}=0$.

We turn now to $\left(\partial^{2}[-S]\right)^{-1}$. Recall equation (4.1.2). Then:

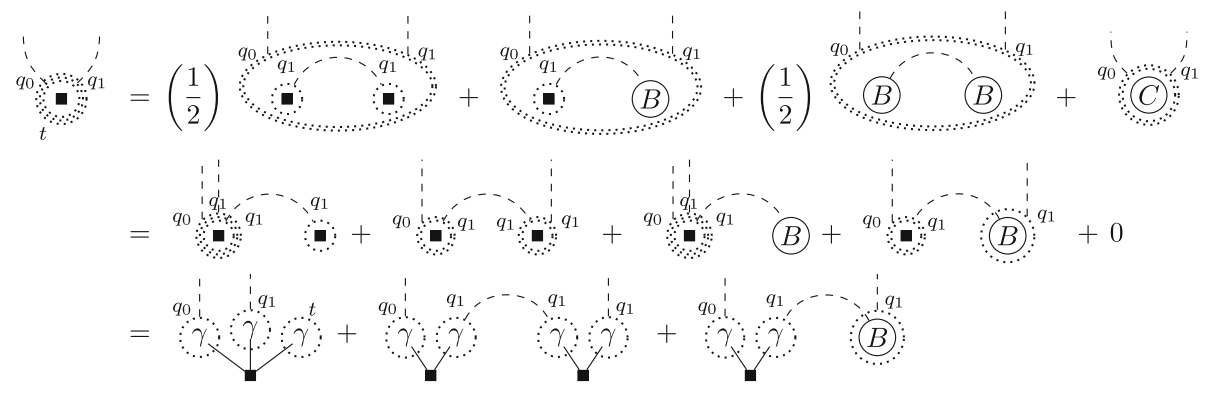


In the second line above we used the product rule and dropped any terms involving derivatives with respect to $q_{0}$ of functions only of $q_{1}$. In the third line we used equations (4.1.3) (4.2.5) and (4.2.6).

By the quotient rule:

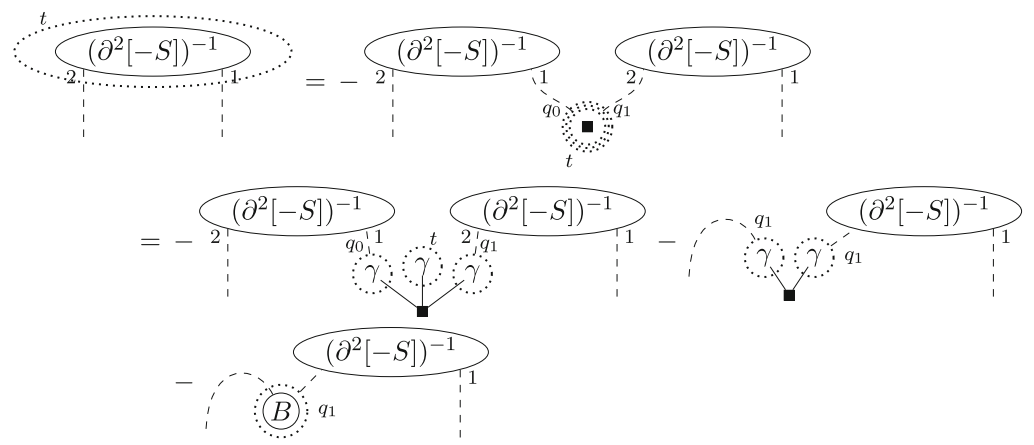

Thus, we can differentiate the Green's function by recalling equation (4.1.4) and using equations(4.3.3) and (4.3.5). Many terms cancel:

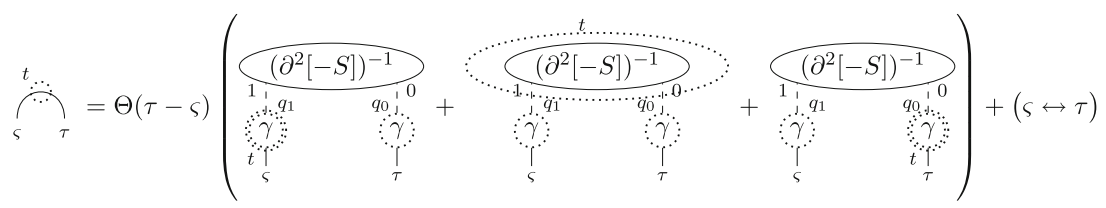

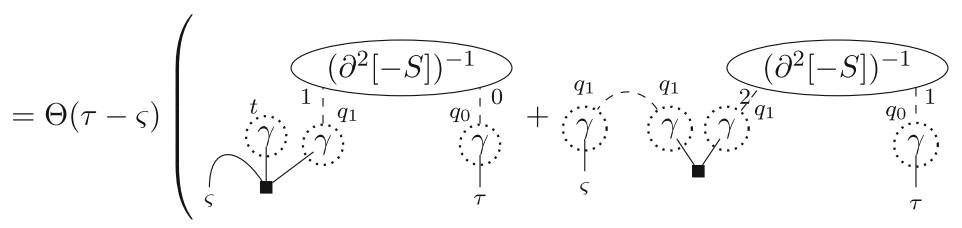

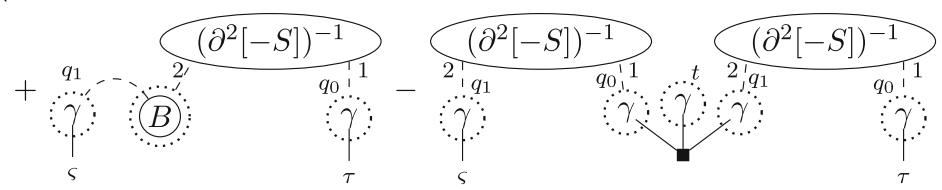

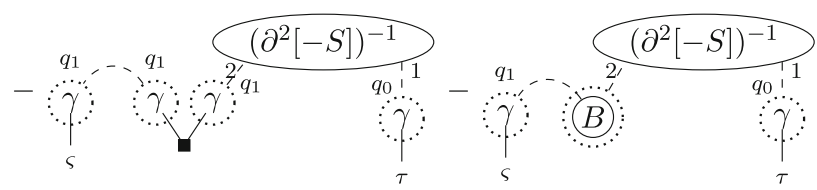

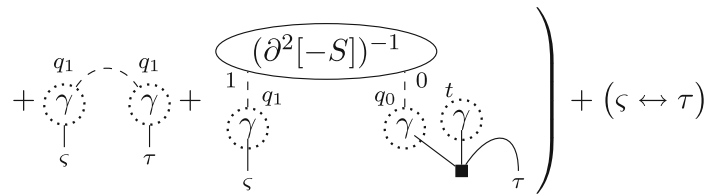




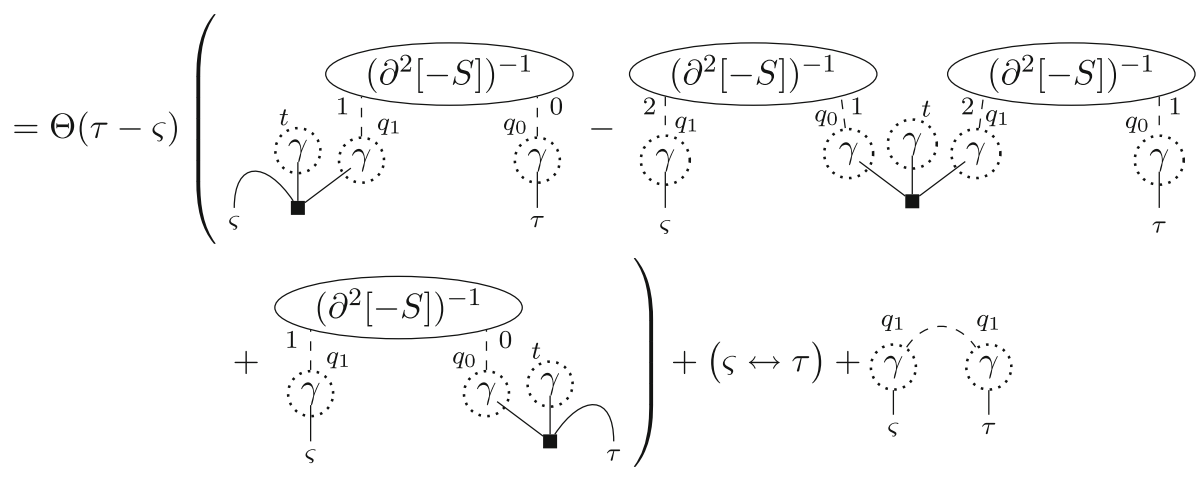

Finally, consider the integrals implicit in the trivalent vertices in equation (4.3.6). Write $\rho$ for the variable of integration for each, and consider the sum as a single integral $\int_{\rho=0}^{t}$. When $\rho \leq \varsigma \leq \tau$, the second and third terms exactly cancel; when $\varsigma \leq$ $\rho \leq \tau$, the three terms agree up to sign; and when $\varsigma \leq \tau \leq \rho$, the first two terms cancel. (When $\tau \leq \varsigma$, the contribution to the total expression is from the part we have abbreviate " $(\varsigma \leftrightarrow \tau)$ ", and the analysis is similar.) But consider breaking the integral $\overbrace{\tau}$ as a similar sum of contributions from $\rho \leq \varsigma \leq \tau$, etc. Equation (4.1.4) implies that the contributions from each part exactly match the integrals in equation (4.3.6). This proves:

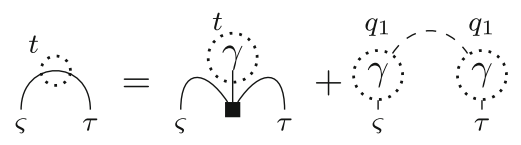

Given equations (4.2.6) and (4.3.4) and the well-known formula for the logarithmic derivative of a determinant, we also have:

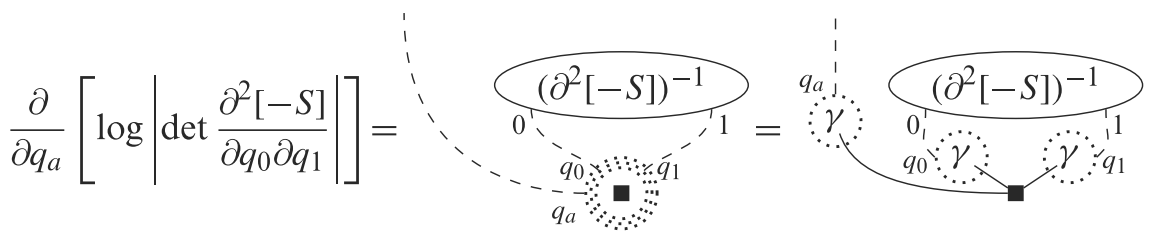

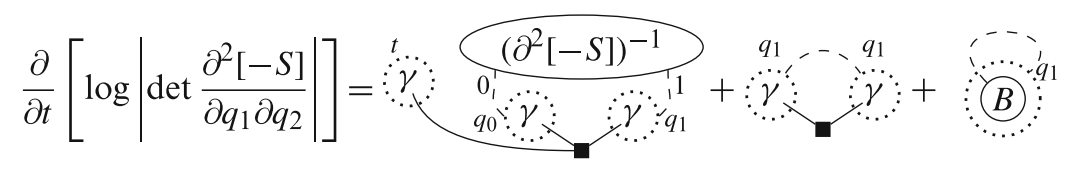

We conclude this section by simplifying the right-hand side of equation (4.3.10) and the first term on the right-hand side of equation (4.3.11). We first expand the 
-vertex:

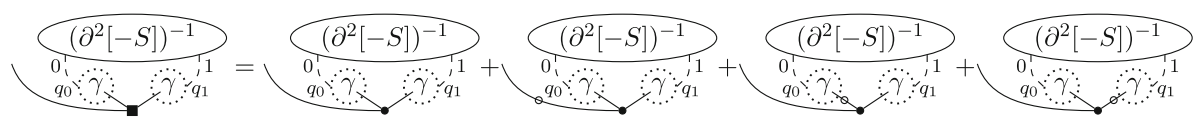

But the integrals implicit in the first two summands above are obviously symmetric under switching the two " $\gamma$ " legs. Recalling equation (4.1.4):

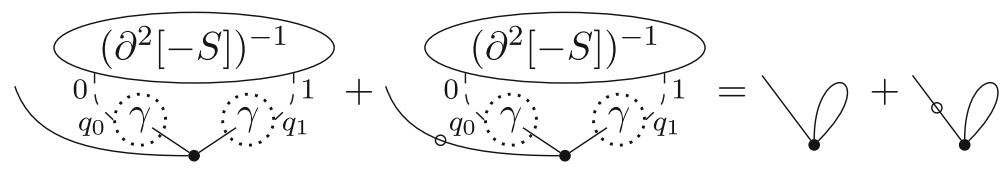

Similarly:

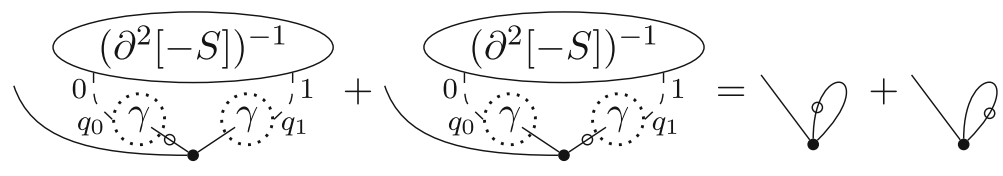

All together, we have the following simplification:

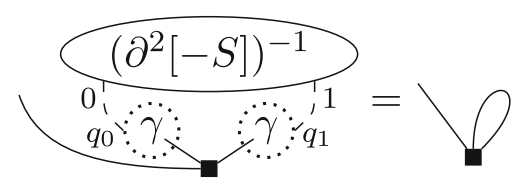

\subsection{THE CANCELLATIONS}

In the previous sections, we evaluated the derivatives of every component of $V_{\gamma}$. In this section, we check equation (4.0.1), which in the graphical notation reads:

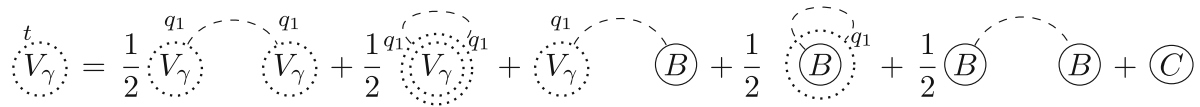

We have adopted the following convention: the dashed lines (although not the dotted circles) count as edges and the new nodes count as vertices for the purposes of computing Euler characteristic, etc., and in equations with connected diagrams with different numbers of loops, we multiply each diagram $\Gamma$ by $(i \hbar)^{\lambda(\Gamma)}$. 
Recalling that $V_{\gamma}=\mathbf{\square}+\left(\frac{i \hbar}{2}\right) \log \left|\operatorname{det} \frac{\partial^{2}\left[-S_{\gamma}\right]}{\partial q_{0} \partial q_{1}}\right|+\sum\{$ connected diagrams $\} /\{$ symmetry $\}$, the calculations from the previous sections give:

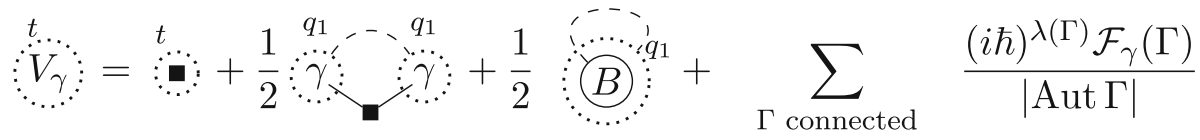

$$
\begin{aligned}
& \text { with one } \stackrel{t}{\because} \because \text { : or } \\
& \text { one } \stackrel{q_{1}}{a} \ddot{\gamma}:--\because \ddot{\gamma}^{q_{1}}
\end{aligned}
$$

In the sum, it is implied that all other vertices are usual $\mathbf{n}$-vertices and are of valence $n \geq 3$. The sum includes the diagram $\operatorname{det} \frac{\partial^{2}\left[-S_{\gamma}\right]}{\partial q_{0} \partial q_{1}} \mid$. Otherwise, each diagram with a ${ }^{t} \dddot{\gamma}:$ comes from the diagram formed by removing it (if the ${ }^{t} \dddot{\gamma}:$ attaches to a trivalent vertex, its removal leaves just a solid edge), and the combinatorics are as in equation (4.1.1): if there are $n$ equiv-

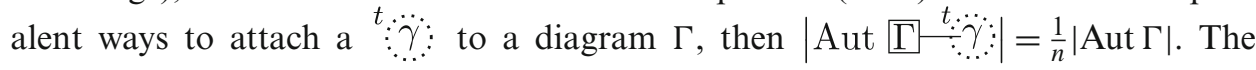
second sum occurs similarly, with each $\stackrel{q_{1}}{\vdots} \dddot{\gamma}:-\cdots q^{q_{1}}$ replacing a Green's function, as per the second term in equation (4.3.9).

Similarly, we have:

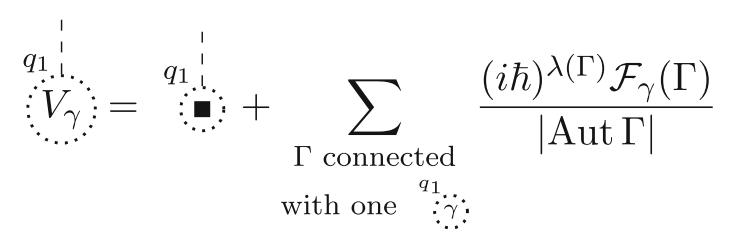

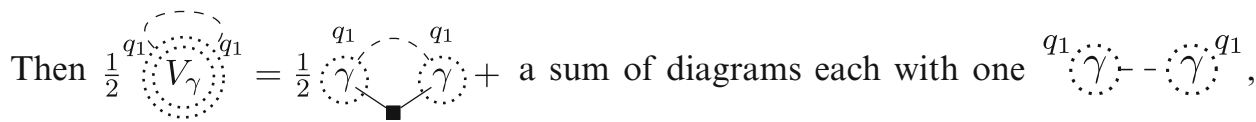
such that its removal yields a diagram that is still connected.

The diagrams in $\frac{1}{2} V_{\gamma}^{q_{1}} V_{\gamma}^{q_{1}}$ come in two types. Other than the simplyconnected diagram $\frac{1}{2} q_{1}^{q_{1}} q_{1}$, there are diagrams where the dashed line connects a complicated part to a $q_{1} \ldots$, and diagrams where the dashed line connects two non-simply-connected parts, which are not connected otherwise. But recall 
equation (4.1.3); then:

$$
\begin{aligned}
& \begin{array}{c}
q_{1}, \cdots q_{1} \\
\frac{q_{2}}{V_{\gamma}} \quad V_{\gamma}+V_{\gamma}
\end{array}
\end{aligned}
$$

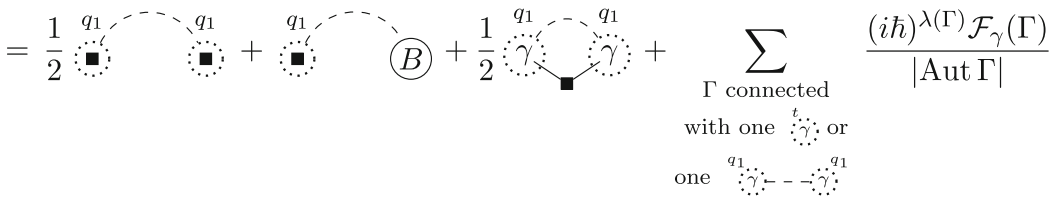

Comparing equations (4.4.1), (4.4.2) and (4.4.4), we see that the only remaining terms are equal by equation (4.1.2). Thus we have proven Theorem 2.2.6.

\section{Proof of Theorem 2.3.3}

Recall that the pointwise convergence of distributions is defined by testing against compactly-supported functions. For a chosen open neighborhood $\mathcal{O} \subseteq \mathbb{R}^{2 n+1}$, let $A$ be the set of all smooth families of classical paths with boundary values in $\mathcal{O}$, and fix $\left(t, q_{1}\right) \in \mathbb{R}^{n+1}$. Throughout this section, we will let $g: \mathbb{R}^{n} \rightarrow \mathbb{R}$ be smooth with compact support $K$ such that $\{t\} \times K \times\left\{q_{1}\right\} \subseteq \mathcal{O}$. We will prove Theorem 2.3.3 in three steps. In Section 5.1, we characterize those families $\gamma \in A$ such that $\int_{K}^{\text {formal }} \exp \left(-(i \hbar)^{-1} V_{\gamma}\left(t, q_{0}, q_{1}\right)\right) g\left(q_{0}\right) \mathrm{d} q_{0}$ is non-zero, and show that there are only finitely many such families. In Section 5.2 we pick $\mathcal{O}_{0} \subseteq \mathbb{R}^{n}$ open with compact closure and show that for $\epsilon>0$ sufficiently small depending on $\mathcal{O}_{0}$, for $\mathcal{O}=(0, \epsilon) \times$ $\mathcal{O}_{0} \times \mathcal{O}_{0}$ there is a unique family of classical paths $\gamma$ with $\int_{K}^{\text {formal }} \exp \left(-(i \hbar)^{-1}\right.$ $\left.V_{\gamma}\left(t, q_{0}, q_{1}\right)\right) g\left(q_{0}\right) \mathrm{d} q_{0} \neq 0$. In Section 5.3 we compute the limit as $t \rightarrow 0$ of this formal integral.

\subsection{ON THE SUPPORT OF FORMAL INTEGRALS}

We wish to understand the formal integral:

$$
\int_{K}^{\text {formal }} \exp \left((i \hbar)^{-1} V_{\gamma}\left(t, q_{0}, q_{1}\right)\right) g\left(q_{0}\right) \mathrm{d} q_{0}
$$

We begin by splitting the exponent $V_{\gamma}=\left(-S_{\gamma}\right)+\left(V_{\gamma}+S_{\gamma}\right)$. Then $V_{\gamma}+S_{\gamma}$ is valued in formal power series that begin in degree $\hbar$, so $\exp \left((i \hbar)^{-1}\left(V_{\gamma}+S_{\gamma}\right)\right)$ is a formal power series. Thus the formal integral in equation (5.1.1) is determined by the $\hbar \rightarrow 0$ asymptotics of integrals of the form $\int_{K} \exp \left(-(i \hbar)^{-1} S_{\gamma}\left(t, q_{0}, q_{1}\right)\right) g\left(q_{0}\right) \mathrm{d} q_{0}$. 
Such integrals are conventionally studied by the method of stationary phase. Indeed, by [4, Lemma 3.13], if $\frac{\partial S_{\gamma}}{\partial q_{0}} \neq 0$ in $K$, then:

$$
\int_{K} \exp \left(-(i \hbar)^{-1} S_{\gamma}\left(t, q_{0}, q_{1}\right)\right) g\left(q_{0}\right) \mathrm{d} q_{0}=O\left(\hbar^{\infty}\right) \text { as } \hbar \rightarrow 0
$$

By equation (2.1.8), $\frac{\partial S_{\gamma}}{\partial q_{0}}\left(t, q_{0}, q_{1}\right)=0$ if and only if $\dot{\gamma}_{\left(t, q_{0}, q_{1}\right)}(0)=-B\left(q_{0}\right)$, where $\gamma_{\left(t, q_{0}, q_{1}\right)}$ is the member of the family of paths $\gamma$ that connects $q_{0}$ to $q_{1}$ in time $t$. If the family $\gamma$ contains a path with initial values $(\dot{\gamma}(0), \gamma(0))=\left(-B\left(q_{0}\right), q_{0}\right)$ for some $q_{0} \in K$, then it does so in an open neighborhood of $q_{0}$, and $\gamma$ is determined on the connected component of $\{t\} \times K \times\left\{q_{1}\right\}$ containing $\left(t, q_{0}, q_{1}\right)$. Thus, as $K$ is compact, there are only finitely many families $\gamma$ with $\frac{\partial S_{\gamma}}{\partial q_{0}}\left(t, q_{0}, q_{1}\right)=0$ for some $q_{0} \in K$, and so there are only finitely many paths $\gamma$ such that the formal integral in equation (5.1.1) is non-zero as a formal expression in $\hbar$.

\subsection{SHORT-DURATION CLASSICAL PATHS CAN BE ALMOST GEODESIC}

For $\epsilon \in \mathbb{R}$, consider the non-degenerate second-order ODE given by:

$$
0=\ddot{\gamma}^{i}+\epsilon\left(\frac{\partial B_{i}}{\partial q^{j}}(\gamma)-\frac{\partial B_{j}}{\partial q^{i}}(\gamma)\right) \dot{\gamma}^{j}+\epsilon^{2} \frac{\partial C}{\partial q^{i}}(\gamma)
$$

Let $\varphi_{\epsilon}$ be the "flow for duration 1" map, i.e. $\varphi_{\epsilon}(v, q)=\left(\dot{\gamma}_{\epsilon}(1), \gamma_{\epsilon}(1)\right)$ for $\gamma_{\epsilon}$ the unique solution to equation (5.2.1) with $\left(\dot{\gamma}_{\epsilon}(0), \gamma_{\epsilon}(0)\right)=(v, q)$. It is defined on an open neighborhood of the 0 section in $\mathrm{TR}^{n}=\mathbb{R}^{2 n}$, it is smooth when it is defined, and it depends smoothly on $\epsilon$. Let $\phi_{\epsilon}(v, q)=\left(q, \pi \circ \varphi_{\epsilon}(v, q)\right)$, where $\pi: \mathbb{T R}^{n} \rightarrow$ $\mathbb{R}^{n}$ is the natural projection. Then $\phi_{0}$ is the isomorphism $(v, q) \mapsto(q, q+v)$. So the partial function $\phi_{[-]}: T \mathbb{R} \times \mathbb{R} \rightarrow \mathbb{R}^{2 n}$ is defined on an open neighborhood of $\mathrm{TR}^{n} \times\{0\}$.

Fix $\mathcal{O}_{0} \subseteq \mathbb{R}^{n}$ with compact closure $\overline{\mathcal{O}_{0}}$, and let $\mathcal{O}_{1} \supseteq \overline{\mathcal{O}_{0}}$ be open with compact closure $\overline{\mathcal{O}_{1}}$. Let $\mathcal{P}=\phi_{0}^{-1}\left(\overline{\mathcal{O}_{1}} \times \overline{\mathcal{O}_{1}}\right)$. It is a compact subset of $\operatorname{TR}^{n}$ containing $\{0\} \times$ $\overline{\mathcal{O}_{1}}$. Then we can find $\epsilon_{0}>0$ such that $\mathcal{P} \times\left(-\epsilon_{0}, \epsilon_{0}\right)$ is contained in the domain of $\phi_{[-]}$. Let $(v, q) \in \mathcal{P}$ such that $q \in \mathcal{O}_{1}$. For $\epsilon \in\left(-\epsilon_{0}, \epsilon_{0}\right)$, let $\gamma_{\epsilon}$ be the duration-1 solution to equation (5.2.1) with initial conditions $\left(\dot{\gamma}_{\epsilon}(0), \gamma_{\epsilon}(0)\right)=(v, q)$. Then $\gamma_{0}$ is nondegenerate, and the nondegeneracy condition depends smoothly on $\epsilon$. Thus for each $(v, q)$ there is some number $0<\epsilon_{1}(v, q)<\epsilon_{0}$ so that for $\epsilon \in\left(-\epsilon_{1}, \epsilon_{1}\right), \gamma_{\epsilon}$ is nondegenerate. By lemma 2.1.4, $\epsilon_{1}$ can be taken to depend lower-semicontinuously on $(v, q)$. Thus it has a minimum value $\epsilon_{2}$ on the compact set $\left\{(v, q) \in \mathcal{P}\right.$ s.t. $\left.q \in \overline{\mathcal{O}_{0}}\right\}$.

Then $\epsilon_{2}>0$ satisfies the following: for each $\epsilon \in\left(-\epsilon_{2}, \epsilon_{2}\right)$ and for each $\left(q_{0}, q_{1}\right) \in$ $\mathcal{O}_{0} \times \mathcal{O}_{0}$, we have chosen a nondegenerate duration-1 solution to equation (5.2.1) connecting $q_{0}$ to $q_{1}$, and the set of all these chosen paths for a given $\epsilon$ is precisely the set of solutions $\gamma_{\epsilon}$ to equation (5.2.1) with $\left(\dot{\gamma}_{\epsilon}(0), \gamma_{\epsilon}(0)\right) \in \mathcal{P}$ and $\gamma(0), \gamma(1) \in$ $\mathcal{O}_{0}$. Thus this family $\gamma_{[-]}$is smooth.

However, if $\epsilon>0$ and $\gamma_{\epsilon}$ is a duration-1 solution to equation (5.2.1), then $\tau \mapsto$ $\gamma_{\epsilon}\left(\epsilon^{-1} \tau\right)$ is a duration- $\epsilon$ solution to equation (2.1.2) (equation (5.2.1) with $\epsilon=1$ ). 
In particular, we have constructed a smooth family $\gamma$ of classical paths with boundary values ranging in $\mathcal{O}_{0} \times \mathcal{O}_{0} \times\left(0, \epsilon_{2}\right)$.

Moreover, suppose that $\gamma$ is a duration- $\epsilon$ solution to equation (2.1.2) with initial conditions $\gamma(0) \in \mathcal{O}_{0}$ and $\dot{\gamma}(0)=-B(\gamma(0))$. Then if $\epsilon$ is sufficiently small depending on $\gamma(0)$, we have $(\epsilon \dot{\gamma}(0), \gamma(0)) \in \mathcal{P}$, and so $\gamma$ is a member of our family. In particular, our family contains all paths with $\dot{\gamma}(0)=-B(\gamma(0))$ that start and end in $\mathcal{O}_{0}$ with sufficiently small duration depending on the endpoints.

\subsection{THE LIMIT AS $t \rightarrow 0$}

In Section 5.1, we showed that the only contributions to an integral of the form

$$
\begin{aligned}
& \int_{K}^{\text {formal }} \exp \left((i \hbar)^{-1} V_{\gamma}\left(t, q_{0}, q_{1}\right)\right) g\left(q_{0}\right) \mathrm{d} q_{0} \\
& =\int_{K}^{\text {formal }} e^{-(i \hbar)^{-1} S_{\gamma}\left(t, q_{0}, q_{1}\right)} \sqrt{\left|\operatorname{det} \frac{\partial^{2}\left[-S_{\gamma}\right]}{\partial q_{0} \partial q_{1}}\right|} \exp \left(\sum \text { diagrams }\right) g\left(q_{0}\right) \mathrm{d} q_{0}
\end{aligned}
$$

come from families $\gamma$ that include paths with initial conditions $\dot{\gamma}(0)=-B(\gamma(0))$. Indeed, for fixed $t, q_{1}$, equation (5.3.1) is supported only at the points $q_{0} \in K$ so that the duration- $t$ path with initial conditions $(\dot{\gamma}(0), \gamma(0))=\left(-B\left(q_{0}\right), q_{0}\right)$ ends at $\gamma(t)=q_{1}$. In Section 5.2 we showed that for $\mathcal{O}=\left(0, \epsilon_{2}\right) \times \mathcal{O}_{0} \times \mathcal{O}_{0}$ there is a unique such family, and that for $q_{1} \in \mathcal{O}_{0}$ and sufficiently small $t$ depending on $q_{1}$, there is a unique such point $q_{0}$. Indeed, we have $q_{1}-q_{0}=-t B\left(q_{0}\right)+O\left(t^{2}\right)$. We remark also that $\exp \left(\sum\right.$ diagrams $)=1+O(\hbar)$.

We claim that for these triples $\left(t, q_{0}, q_{1}\right)$, we have:

$$
\left(\left(\frac{\partial^{2}\left[-S_{\gamma}\right]}{\partial q_{1} \partial q_{0}}\right)^{-1}\right)^{k l}=t \delta^{k l}+O\left(t^{2}\right)
$$

Indeed, consider integrating equation (2.1.2) twice, with initial conditions $(\dot{\gamma}(0)$, $\gamma(0))=\left(v_{0}, q_{0}\right)$ :

$$
\gamma^{i}(t)=q_{0}^{i}+t v_{0}^{i}+\left.\int_{0}^{t} \int_{0}^{\tau}\left(\left(\frac{\partial B_{k}}{\partial q^{i}}-\frac{\partial B_{i}}{\partial q^{k}}\right) \dot{\gamma}^{k}(\varsigma)-\frac{\partial C}{\partial q^{i}}\right)\right|_{q=\gamma(\varsigma)} \mathrm{d} \varsigma \mathrm{d} \tau
$$

Then holding $q_{0}$ fixed we have:

$$
\begin{aligned}
\frac{\partial \gamma^{i}(t)}{\partial v_{0}^{j}}= & t \delta_{j}^{i}+\int_{0}^{t} \int_{0}^{\tau}\left(\frac{\partial}{\partial q^{l}}\left(\frac{\partial B_{k}}{\partial q^{i}}-\frac{\partial B_{i}}{\partial q^{k}}\right) \frac{\partial \gamma^{l}(\varsigma)}{\partial v_{0}^{j}} \dot{\gamma}^{k}(\varsigma)\right. \\
& \left.+\left(\frac{\partial B_{k}}{\partial q^{i}}-\frac{\partial B_{i}}{\partial q^{k}}\right) \frac{\partial \dot{\gamma}^{k}(\varsigma)}{\partial v_{0}^{j}}-\frac{\partial}{\partial q^{l}}\left(\frac{\partial C}{\partial q^{i}}\right)\right)\left.\right|_{q=\gamma(\varsigma)} \mathrm{d} \varsigma \mathrm{d} \tau
\end{aligned}
$$


We now take $t$ to be sufficiently small and $v_{0}$ to vary only in a compact neighborhood of $-B\left(q_{0}\right)$. Then $\frac{\partial \gamma(\varsigma)}{\partial v_{0}}, \frac{\partial \dot{\gamma}(\varsigma)}{\partial v_{0}}=O(1)$ as $t \rightarrow 0$, but then equation (5.3.3) gives $\frac{\partial \gamma^{i}(t)}{\partial v_{0}^{j}}=t \delta_{j}^{i}+O\left(t^{2}\right)$. On the other hand, as we observed in equation (3.2.2), it follows from equation (2.1.8) that $\left(\frac{\partial^{2}\left[-S_{\gamma}\right]}{\partial q_{1} \partial q_{0}}\right)^{-1}=\left(\frac{\partial v_{0}}{\partial q_{1}}\right)^{-1}=\frac{\partial q_{1}}{\partial v_{0}}$. This gives equation (5.3.2).

We make a similar argument, now holding $q_{1}$ fixed:

$$
\begin{aligned}
q_{0}^{i}=q_{1}^{i}-t v_{0}^{i}-\left.\int_{0}^{t} \int_{0}^{\tau}\left(\left(\frac{\partial B_{k}}{\partial q^{i}}-\frac{\partial B_{i}}{\partial q^{k}}\right) \dot{\gamma}^{k}(\varsigma)-\frac{\partial C}{\partial q^{i}}\right)\right|_{q=\gamma(\varsigma)} \mathrm{d} \varsigma \mathrm{d} \tau \\
\frac{\partial q_{0}^{i}}{\partial v_{0}^{j}}=-t \delta_{j}^{i}+O\left(t^{2}\right) \\
\left(\left(\frac{\partial^{2}\left[S_{\gamma}\right]}{\left(\partial q_{0}\right)^{2}}\right)^{-1}\right)^{k l}=\left(\left(-\frac{\partial}{\partial q_{0}}\left(v_{0}+B\left(q_{0}\right)\right)\right)^{-1}\right)^{k l} \\
=\left(\left(t^{-1} \delta+O(1)\right)^{-1}\right)^{k l}=t \delta^{k l}+O\left(t^{2}\right)
\end{aligned}
$$

Then, by [4, Theorem 3.15(ii)], we have:

$$
\begin{aligned}
& \quad \int^{\text {formal }} \exp \left((i \hbar)^{-1} V_{\gamma}\left(t, q_{0}, q_{1}\right)\right) g\left(q_{0}\right) \mathrm{d} q_{0} \\
& =(2 \pi \hbar)^{n / 2} \times e^{i n \pi / 4} \times \operatorname{det}\left(t \delta_{j}^{i}+O\left(t^{2}\right)\right)^{-1 / 2} \times e^{-(i \hbar)^{-1} S_{\gamma}\left(t, q_{1}+B\left(q_{1}\right)+O\left(t^{2}\right), q_{1}\right)} \\
& \quad \times \operatorname{det}\left(t \delta_{j}^{i}+O\left(t^{2}\right)\right)^{1 / 2} \times g\left(q_{1}+B\left(q_{1}\right)+O\left(t^{2}\right)\right) \times(1+O(\hbar))
\end{aligned}
$$

But $S_{\gamma}\left(t, q_{1}+B\left(q_{1}\right)+O\left(t^{2}\right), q_{1}\right)=\int_{0}^{t}\left(\frac{1}{2}|\dot{\gamma}|^{2}+B(\gamma) \cdot \dot{\gamma}-C(\gamma)\right) \mathrm{d} \tau=\int_{0}^{t}\left(\frac{1}{2}(\dot{\gamma}+B)^{2}+\right.$ $O(1)) \mathrm{d} \tau=\int_{0}^{t}(O(t)+O(1)) \mathrm{d} \tau=O(t)$. Thus:

$$
\lim _{t \rightarrow 0} \int^{\text {formal }} \exp \left((i \hbar)^{-1} V_{\gamma}\left(t, q_{0}, q_{1}\right)\right) g\left(q_{0}\right) \mathrm{d} q_{0}=(2 \pi \hbar)^{n / 2} e^{i n \pi / 4}\left(g\left(q_{1}\right)+O(\hbar)\right)
$$

What about the order- $\hbar$ terms? They can be given explicitly as a sum along the lines of our equation (2.2.7) — c.f. [14,15,8] — or implicitly as in [4]. All we need is the following fact: each degree in $\hbar$ is given by a finite sum in which for each summand there is some $M$ so that the summand scales as $\left(\frac{\partial^{2}\left[-S_{\gamma}\right]}{\left(\partial q_{0}\right)^{2}}\right)^{-M}$ times a product of strictly fewer than $M$ terms given by derivatives of $g$ and $V_{\gamma}$ at $q_{0}=q_{1}+O\left(t^{2}\right)$.

The calculations in Section 4 in fact allow us to write down all derivatives of $V_{\gamma}$ with respect to $q_{0}$. In addition to the derivatives computed in Section 4.4, we 
have:

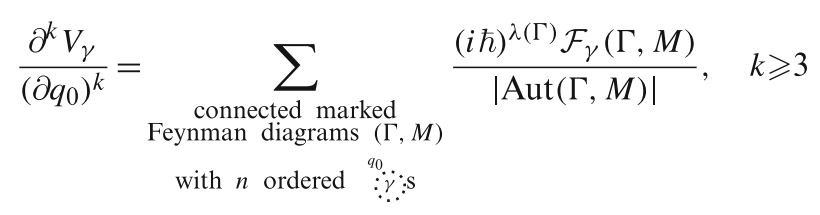

Consider expanding each edge as in equation (4.1.4). Then in each diagram a marked half edge converts some $\frac{\partial \gamma}{\partial q_{a}}$ into $\frac{\partial \dot{\gamma}}{\partial q_{a}}=-B\left(q_{0}\right)+O(t)$. On the other hand, each vertex corresponds to an integration $\int_{0}^{t}$ of bounded functions, and so the vertices are $O(t)$. Thus, by equation (5.3.2), $\mathcal{F}_{\gamma}(\Gamma, M)=O\left(t^{|V|+|E|}\right)$ for each diagram $\Gamma$ in equation (5.3.8). As the number of diagrams at each order is finite, this proves that for $k \geq 3, \frac{\partial^{k} V_{\gamma}}{\partial q_{0}^{k}}=O(t)$.

But the derivatives of $g$ do not depend on $t ; \frac{\partial V_{\gamma}}{\partial q_{0}}=-\frac{\partial S_{\gamma}}{\partial q_{0}}+\sum\{$ diagrams $\}=0+$ $\sum\{$ diagrams $\}=O(t)$; and $\frac{\partial^{2} V_{\gamma}}{\left(\partial q_{0}\right)^{2}}=-\frac{\partial^{2} S_{\gamma}}{\left(\partial q_{0}\right)^{2}}+\sum\{$ diagrams $\}=O\left(t^{-1}\right)$. As each term after the first one in the asymptotic expansion of equation (5.3.7) has strictly more occurrences of $\left(\frac{\partial^{2}\left[-S_{\gamma}\right]}{\left(\partial q_{0}\right)^{2}}\right)^{-1}$ than of derivatives of $g$ and $V_{\gamma}$, we see that the $O(\hbar)$ part of equation (5.3.7) is also $O(t)$. Thus, as formal expressions in $\hbar$ :

$$
\lim _{t \rightarrow 0} \int^{\text {formal }} \exp \left((i \hbar)^{-1} V_{\gamma}\left(t, q_{0}, q_{1}\right)\right) g\left(q_{0}\right) \mathrm{d} q_{0}=(2 \pi \hbar)^{n / 2} e^{i n \pi / 4} g\left(q_{1}\right)
$$

Theorem 2.3.3 is proved.

\section{Acknowledgements}

This project was suggested by N. Reshetikhin, who provided support and suggestions throughout all stages of it. K. Datchev, C. Schommer-Pries, G. Thompson, and I. Ventura provided valuable discussions. I would like to also thank the anonymous referee for alerting me to the work of $\mathrm{H}$. Kleinert and collaborators. I am grateful to Aarhus University for the hospitality. This work is supported by NSF grant DMS-0901431.

Open Access This article is distributed under the terms of the Creative Commons Attribution Noncommercial License which permits any noncommercial use, distribution, and reproduction in any medium, provided the original author(s) and source are credited.

\section{References}

1. Albeverio, S., Höegh-Krohn, R.: Oscillatory integrals and the method of stationary phase in infinitely many dimensions, with applications to the classical limit of quantum mechanics, I. Invent. Math. 40(1), 59-106 (1977)

2. DeWitt-Morette, C.: The semiclassical expansion. Ann. Phys. 97(2), 367-399 (1976) 
3. Duru, I.H., Kleinert, H.: Quantum mechanics of H-atom from path integrals. Fortschr. Phys. 30(8), 401-435 (1982)

4. Evans, L.C., Zworski, M.: Lectures on semiclassical analysis. Available at http://math. berkeley.edu/ $\sim$ zworski/semiclassical.pdf (2007)

5. Feynman, R.P.: Space-time approach to non-relativistic quantum mechanics. Rev. Mod. Phys. 20(2), 367-387 (1948)

6. Feynman, R.P., Hibbs, A.R.: Quantum Mechanics and Path Integrals. International Series in Pure and Applied Physics. McGraw-Hill, New York (1965)

7. Johnson, G.W., Lapidus, M.L.: The Feynman Integral and Feynman's Operational Calculus. Oxford Mathematical Monographs. The Clarendon Press Oxford University Press, New York. Oxford Science Publications (2000)

8. Johnson-Freyd, T.: The formal path integral and quantum mechanics. J. Math. Phys. Available at http://math.berkeley.edu/ $\sim$ theojf/QM3.pdf or arXiv:1004.4305 [math-ph] (in press)

9. Johnson-Freyd, T., Schommer-Pries, C.: Critical points on a fiber bundle. Online forum discussion at http://mathoverflow.net/questions/134/ (2009)

10. Klauder, J.R., Daubechies, I.: Quantum mechanical path integrals with Wiener measures for all polynomial Hamiltonians. Phys. Rev. Lett. 52(14), 1161-1164 (1984)

11. Kleinert, H., Chervyakov, A.: Integrals over products of distributions from perturbation expansions of path integrals in curved space. Int. J. Mod. Phys. A 17(15), 20192050 (2002)

12. Manuel, C., Tarrach, R.: Perturbative renormalization in quantum mechanics. Phys. Lett. B 328, 113-118 (1994)

13. Milnor, J.: Morse theory. Based on lecture notes by M. Spivak and R. Wells. Annals of Mathematics Studies, No. 51. Princeton University Press, Princeton (1963)

14. Polyak, M.: Feynman diagrams for pedestrians and mathematicians. In: Lyubich, M., Takhtajan, L.A. (eds.) Graphs and Patterns in Mathematics and Theoretical Physics. Proc. Sympos. Pure Math., vol. 73, pp. 15-42. American Mathematical Society, Providence (2005)

15. Reshetikhin, N.: Lectures on Quantization of Gauge Systems. ArXiv e-prints, arXiv: 1008.1411 [math-ph] (2010)

16. Takhtajan, L.A.: Quantum Mechanics for Mathematicians. American Mathematical Society, Providence (2008) 